DOI 10.4171/JEMS/335

Oana Ivanovici

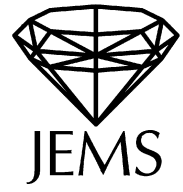

\title{
Counterexamples to the Strichartz inequalities for the wave equation in general domains with boundary
}

Received August 4, 2010 and in revised form December 13, 2010

\begin{abstract}
We consider a smooth and bounded domain $\Omega \subset \mathbb{R}^{d}$ of dimension $d \geq 2$ with boundary and we construct sequences of solutions to the wave equation with Dirichlet boundary condition which fail the Strichartz estimates of the free space, providing losses of derivatives at least for a subset of the usual range of indices. This is due to microlocal phenomena such as caustics generated in arbitrarily small time near the boundary. Moreover, the result holds for microlocally strictly convex domains in $\mathbb{R}^{d}$.
\end{abstract}

Keywords. Microlocal analysis, wave equation, Dirichlet boundary condition, Strichartz estimates, propagation and reflection of singularities, conormal waves with cusps, caustics

\section{Introduction}

Let $\Omega$ be a smooth manifold of dimension $d \geq 2$ with $C^{\infty}$ boundary $\partial \Omega$, equipped with a Riemannian metric $g$. Let $\Delta_{g}$ be the Laplace-Beltrami operator associated to $g$ on $\Omega$, acting on $L^{2}(\Omega)$ with Dirichlet boundary condition. Let $0<T<\infty$ and consider the wave equation with Dirichlet boundary conditions:

$$
\left\{\begin{array}{l}
\left(\partial_{t}^{2}-\Delta_{g}\right) u=0 \quad \text { on } \Omega \times[0, T] \\
\left.u\right|_{t=0}=u_{0},\left.\quad \partial_{t} u\right|_{t=0}=u_{1}, \\
\left.u\right|_{\partial \Omega}=0
\end{array}\right.
$$

Strichartz estimates are a family of dispersive estimates on solutions $u: \Omega \times[0, T] \rightarrow \mathbb{C}$ to the wave equation (1.1). In their most general form, local Strichartz estimates state that

$$
\|u\|_{L^{q}\left([0, T], L^{r}(\Omega)\right)} \leq C\left(\left\|u_{0}\right\|_{\dot{H}^{\gamma}(\Omega)}+\left\|u_{1}\right\|_{\dot{H}^{\gamma-1}}\right),
$$

where $\dot{H}^{\gamma}(\Omega)$ denotes the homogeneous Sobolev space over $\Omega$ and where the pair $(q, r)$ is wave admissible in dimension $d$, i.e. $2 \leq q \leq \infty, 2 \leq r<\infty$ and

$$
\frac{1}{q}+\frac{d}{r}=\frac{d}{2}-\gamma, \quad \frac{2}{q}+\frac{d-1}{r} \leq \frac{d-1}{2} .
$$

O. Ivanovici: Department of Mathematics J. A. Dieudonné, University of Nice Sophia-Antipolis, Parc Valrose, 06108 Nice Cedex 02, France; e-mail: Oana.Ivanovici@ math.unice.fr

Mathematics Subject Classification (2010): Primary 35L20; Secondary 58J30, 58 J32 
When equality holds in (1.3) the pair $(q, r)$ is called sharp wave admissible in dimension $d$. Estimates involving $r=\infty$ hold when $(q, r, d) \neq(2, \infty, 3)$, but typically require the use of Besov spaces.

Our main result is work in the opposite direction. Roughly speaking, we show that if $\Omega \subset \mathbb{R}^{d}$ is a smooth and bounded domain of $\mathbb{R}^{d}$ and $(q, r)$ is a sharp wave admissible pair in dimension $d \geq 2$ with $r>4$, then there exists $T=T(\Omega)$ such that the quotient

$$
\frac{\|u\|_{L^{q}\left([0, T], L^{r}(\Omega)\right)}}{\left\|u_{0}\right\|_{\dot{H}^{\gamma+\frac{1}{6}\left(\frac{1}{4}-\frac{1}{r}\right)-\epsilon}(\Omega)}+\left\|u_{1}\right\|_{\dot{H}^{\gamma+\frac{1}{6}\left(\frac{1}{4}-\frac{1}{r}\right)-1-\epsilon}(\Omega)}}
$$

takes arbitrarily large values for suitable initial data $\left(u_{0}, u_{1}\right)$, which means that a loss of at least $\frac{1}{6}\left(\frac{1}{4}-\frac{1}{r}\right)-\epsilon$ derivatives is unavoidable in the Strichartz estimates for the flow.

The main motivation for the above types of Strichartz estimates comes from applications to harmonic analysis and the study of nonlinear dispersive equations. Estimates like (1.2) can be used to prove existence theorems for nonlinear wave equations.

In $\mathbb{R}^{d}$ and for $g_{i j}=\delta_{i j}$, Strichartz estimates in the context of the wave and Schrödinger equations have a long history, beginning with Strichartz's pioneering work [25], where he proved the particular case $q=r$ for the wave and (classical) Schrödinger equation. This was later generalized to mixed $L^{q}\left((-T, T), L^{r}(\Omega)\right)$ norms by Ginibre and Velo [7] for the Schrödinger equation, where $(q, r)$ is sharp admissible and $q>2$; the wave estimates were obtained independently by Ginibre-Velo [8] and Lindblad-Sogge [17], following earlier work by Kapitanski [13]. The remaining endpoints for both equations were finally settled by Keel and Tao [15]. In that case $\gamma=\frac{d+1}{2}\left(\frac{1}{2}-\frac{1}{r}\right)$ and one can obtain a global estimate with $T=\infty$ (see also Kato [14] and Cazenave-Weissler [5]).

Let us recall the result for the flat space: if $\Delta$ is the Euclidian Laplace operator, then the Strichartz estimates for the wave equation on $\mathbb{R}^{d}$ read as follows (see [15]):

Proposition 1.1. Let $(q, r)$ be a wave admissible pair in dimension $d \geq 2$. If $u$ satisfies

$$
\left(\partial_{t}^{2}-\Delta\right) u=0 \quad \text { on }[0, T] \times \mathbb{R}^{d},\left.\quad u\right|_{t=0}=u_{0},\left.\quad \partial_{t} u\right|_{t=0}=u_{1}
$$

for some $0<T<\infty, u_{0}, u_{1} \in C^{\infty}\left(\mathbb{R}^{d}\right)$, then there is a constant $C=C_{T}$ such that

$$
\|u\|_{L^{q}\left([0, T], L^{r}\left(\mathbb{R}^{d}\right)\right)} \leq C\left(\left\|u_{0}\right\|_{\dot{H}^{\frac{d+1}{2}\left(\frac{1}{2}-\frac{1}{r}\right)}\left(\mathbb{R}^{d}\right)}+\left\|u_{1}\right\|_{\dot{H}^{\frac{d+1}{2}\left(\frac{1}{2}-\frac{1}{r}\right)-1}\left(\mathbb{R}^{d}\right)}\right) .
$$

In the variable coefficients case, even without boundary, the situation is much more complicated: we simply recall here the pioneering work of Staffilani and Tataru [24], dealing with compact, nontrapping perturbations of the flat metric and recent work of Bouclet and Tzvetkov [2] in the context of the Schrödinger equation, which considerably weakens the decay of the perturbation (retaining the nontrapping character at spatial infinity). On compact manifolds without boundary, due to the finite speed of propagation, it is enough to work in coordinate charts and to establish local Strichartz estimates for variable coefficients wave operators in $\mathbb{R}^{d}$ : we recall here the works by Kapitanski [12] and Mockenhaupt, Seeger and Sogge [19] in the case of smooth coefficients when one can use the Lax parametrix construction to obtain the appropriate dispersive estimates. In the case of $C^{1,1}$ coefficients, Strichartz estimates were shown in the works by Smith [21] and by 
Tataru [26], the latter work establishing the full range of local estimates; here the lack of smoothness prevents the use of Fourier integral operators and instead wave packets and coherent state methods are used to construct parametrices for the wave operator. In these situations, if the metric is sufficiently smooth (if it has at least two derivatives bounded), the Strichartz estimates hold as in the Euclidian case.

Even though the case without boundary has been well understood for some time, obtaining results for manifolds with boundary has been surprisingly elusive.

For a manifold with smooth, strictly geodesically concave boundary (i.e. for which the second fundamental form is strictly negative definite), the Melrose and Taylor parametrix yields the Strichartz estimates for the wave equation with Dirichlet boundary condition for the range of exponents in (1.3) (not including the endpoints), as shown in the paper of Smith and Sogge [22]. If the concavity assumption is removed, however, the presence of multiply reflecting geodesic and their limits, the gliding rays, prevents the construction of a similar parametrix.

Note that on an exterior domain a source point does not generate caustics and that the presence of caustics generated in small time near a source point is what makes things difficult inside a strictly convex set.

Recently, Burq, Lebeau and Planchon [3], [4] established Strichartz type inequalities on a manifold with boundary using the $L^{r}(\Omega)$ estimates for the spectral projectors obtained by Smith and Sogge [23]. The range of triples $(q, r, \gamma)$ that can be obtained in this manner, however, is restricted by the allowed range of $r$ in the square function estimate for the wave equation, which controls the norm of $u$ in the space $L^{r}\left(\Omega, L^{2}(-T, T)\right.$ ) (see [23]). In dimension 3, for example, this restricts the indices to $q, r \geq 5$. The work of Blair, Smith and Sogge [1] expands the range of indices $q$ and $r$ obtained in [3]: specifically, they show that if $\Omega$ is a compact manifold with boundary and $(q, r, \gamma)$ is a triple satisfying the first condition in (1.3) together with the restriction

$$
\begin{cases}\frac{3}{q}+\frac{d-1}{r} \leq \frac{d-1}{2}, & d \leq 4, \\ \frac{1}{q}+\frac{1}{r} \leq \frac{1}{2}, & d \geq 4,\end{cases}
$$

then the Strichartz estimates (1.2) hold true for solutions $u$ to (1.1) satisfying Dirichlet or Neumann homogeneous boundary conditions, with a constant $C$ depending on $\Omega$ and $T$.

In this paper we prove that Strichartz estimates for the wave equation inside the domain $\Omega$ suffer losses when compared to the usual case of $\mathbb{R}^{d}$, at least for a subset of the usual range of indices, under our assumption that there exists a point in $T^{*} \partial \Omega$ where the second fundamental form on the boundary of the manifold has a strictly positive eigenfunction. Precisely, our assumption reads as follows:

Assumption 1.2. Let $\Omega$ be a smooth manifold of dimension $d \geq 2$ with $C^{\infty}$ boundary $\partial \Omega$. We assume that there exists a bicharacteristic that intersects $\partial \Omega \times \mathbb{R}$ tangentially at some point $\left(\rho_{0}, \vartheta_{0}\right) \in T^{*}(\partial \Omega \times \mathbb{R})$ having exactly second order contact with the boundary at $\left(\rho_{0}, \vartheta_{0}\right)$ and which remains in the complement of $\bar{\Omega} \times \mathbb{R}$. We call the point $\left(\rho_{0}, \vartheta_{0}\right)$ a gliding point. 
Remark 1.3. In particular, any smooth and bounded domain of $\mathbb{R}^{d}, d \geq 2$, satisfies the condition in Assumption 1.2.

Our main result reads as follows:

Main Theorem 1.4. Let $(\Omega, g)$ satisfy the conditions in Assumption 1.2 with $d \in\{2,3,4\}$. Then there exists $T=T(\Omega) \in(0, \infty)$ and for every small $\epsilon>0$ there exist sequences $V_{h, j, \epsilon} \in C^{\infty}(\bar{\Omega}), j \in\{0,1\}$, such that the solution $V_{h, \epsilon}$ to the wave equation with Dirichlet boundary conditions

$$
\left\{\begin{array}{l}
\left(\partial_{t}^{2}-\Delta_{g}\right) V_{h, \epsilon}=0, \\
\left.V_{h, \epsilon}\right|_{t=0}=V_{h, 0, \epsilon},\left.\quad \partial_{t} V_{h, \epsilon}\right|_{t=0}=V_{h, 1, \epsilon}, \\
\left.V_{h, \epsilon}\right|_{\partial \Omega \times[0, T]}=0,
\end{array}\right.
$$

satisfies

$$
\sup _{\epsilon>0, h \in(0,1], j} h^{-\frac{d+1}{2}\left(\frac{1}{2}-\frac{1}{r}\right)-\frac{1}{6}\left(\frac{1}{4}-\frac{1}{r}\right)+2 \epsilon+j}\left\|V_{h, j, \epsilon}\right\|_{L^{2}(\Omega)} \leq 1
$$

and

$$
\lim _{h \rightarrow 0}\left\|V_{h, \epsilon}\right\|_{L_{t}^{q}\left([0, T], L^{r}(\Omega)\right)}=\infty
$$

for every sharp wave admissible pair $(q, r)$ in dimension $d$ with $r>4$. Moreover $V_{h, \epsilon}$ has compact support for the normal variable in a neighborhood of the boundary of size $h^{(1-\epsilon) / 2}$ and is well localized at spatial frequency $1 / h$ in the tangential variable.

Remark 1.5. Notice that Theorem 1.4 shows an explicit loss of at least $\frac{1}{6}\left(\frac{1}{4}-\frac{1}{r}\right)$ derivatives in the Strichartz estimates for domains $\Omega$ satisfying Assumption 1.2 compared to the Euclidian case (see Proposition 1.1).

Remark 1.6. The proof of Theorem 1.4 will show that the restriction on the dimension comes only from the fact that for $d \geq 5$ all admissible pairs $(q, r)$ satisfy $r \leq 4$.

Remark 1.7. From Remarks 1.3 and 1.6 it follows that Theorem 1.4 holds true if $\Omega$ is any smooth, bounded domain in $\mathbb{R}^{d}$ with $d \in\{2,3,4\}$.

Remark 1.8. In [10] we proved Theorem 1.4 in the particular case of the two-dimensional half-space $\{(x, y) \mid x>0, y \in \mathbb{R}\}$ with Laplace operator given by $\partial_{x}^{2}+(1+x) \partial_{y}^{2}$. We notice that the half-space together with the metric inherited from the above Laplace operator becomes a strictly convex domain (called Friedlander's model domain).

In this paper we generalize the result of [10] to any smooth domain satisfying Assumption 1.2 using Melrose's theorem on glancing surfaces.

Remark 1.9. Notice that Theorem 1.4 states for instance that the scale-invariant Strichartz estimates fail for $3 / q+1 / r>15 / 24$, whereas the result of Blair, Smith and Sogge states that such estimates hold if $3 / q+1 / r \leq 1 / 2$. Of course, the counterexample places a lower bound on the loss for such indices $(q, r)$, and the work [1] would place some 
upper bounds, but this concise statement shows one explicit gap in our knowledge that remains to be filled.

A very interesting and natural question would be to determine the sharp range of exponents for (1.2) in any dimension $d \geq 2$.

A classical way to prove Strichartz inequalities is to use dispersive estimates: the fact that weakened dispersive estimates can still imply optimal (and scale invariant) Strichartz estimates for the solution of the wave equation was first noticed by Lebeau who announced in [16] that a loss of derivatives is unavoidable for the wave equation inside a strictly convex domain, and this appears because of swallowtail type caustics in the wave front set of $u$ :

$$
\left|\chi\left(h D_{t}\right) u(t, x)\right| \lesssim h^{-d} \min \left(1,(h / t)^{\frac{d-2}{2}+\frac{1}{4}}\right) .
$$

As shown in [11] (where a detailed proof of (1.8) is given), the estimates (1.8), although optimal for the dispersion, imply Strichartz type inequalities without losses, but with indices $(q, r, d)$ satisfying

$$
\frac{1}{q} \leq\left(\frac{d-2}{2}+\frac{1}{4}\right)\left(\frac{1}{2}-\frac{1}{r}\right)
$$

In dimension $d \geq 3$ and in the case of strictly convex domains, this range of indices generalizes the one in (1.4) obtained in [1].

In the proof of Theorem 1.4 we use conormal waves with multiply reflected cusps at the boundary, together with Melrose's theorem on glancing rays, to reduce the study of the iterated boundary operators to Friedlander's model, and hence to [10]. The construction of a parametrix for (1.1) is in the same spirit as in the classical paper by J. Ralston [20], which however deals with a very different geometric situation.

The organization of the paper is as follows: In Section 2 we show that in order to prove Theorem 1.4 it is enough to consider the two-dimensional case. In Section 3 we recall the construction in the model case of a strictly convex domain of dimension two we dealt with in [10] and use it to determine an approximate solution of (1.5) which satisfies the conclusion of Theorem 1.4.

\section{Reduction to the two-dimensional case}

Let $\Omega$ satisfy the assumptions of Theorem 1.4. Write local coordinates on $\Omega$ as $\left(x, y_{1}, \ldots, y_{d-1}\right)$ with $x>0$ on $\Omega, \partial \Omega=\left\{(0, y) \mid y=\left(y_{1}, \ldots, y_{d-1}\right) \in \mathbb{R}^{d-1}\right\}$, and local coordinates induced by the product $X=\Omega \times \mathbb{R}_{t}$ as $(x, y, t)$.

Local coordinates on the base induce local coordinates on the cotangent bundle, namely $(\rho, \vartheta)=(x, y, t, \xi, \eta, \tau)$ on $T^{*} X$ near $\pi^{-1}(q), q \in T^{*} \partial X$, where $\pi$ : $T^{*} X \rightarrow{ }^{b} T^{*} X$ is the canonical inclusion from the cotangent bundle into the $b$-cotangent bundle defined by ${ }^{b} T^{*} X=T^{*} \stackrel{\circ}{X} \cup T^{*} \partial X$. The corresponding local coordinates on the 
boundary are denoted $(y, t, \eta, \tau)$ (on a neighborhood of a point $q$ in $T^{*} \partial X$ ). The metric function in $T^{*} \Omega$ has the form

$$
g(x, y, \xi, \eta)=A(x, y) \xi^{2}+2 \sum_{j=1}^{d-1} C_{j}(x, y) \xi \eta_{j}+\sum_{j, k=1}^{d-1} B_{j, k}(x, y) \eta_{j} \eta_{k},
$$

with $A, B_{j, k}, C_{j}$ smooth. Moreover, these coordinates can be chosen so that $A(x, y)=1$ and $C_{j}(x, y)=0$ (see [9, Appendix C]). Thus, in this coordinate chart the metric on the boundary reads

$$
g(0, y, \xi, \eta)=\xi^{2}+\sum_{j, k=1}^{d-1} B_{j, k}(0, y) \eta_{j} \eta_{k}
$$

On $T^{*} \partial \Omega$ the metric $g$ takes an even simpler form, since introducing geodesic coordinates we can assume moreover that, locally,

$$
B_{1,1}(0, y)=1, \quad B_{1, j}(0, y)=0 \quad \forall j \in\{2, \ldots, d-1\} .
$$

Hence, if we write $R(x, y, \eta):=\sum_{j, k=1}^{d-1} B_{j, k}(x, y) \eta_{j} \eta_{k}$, then for small $x$ we have

$$
\begin{aligned}
R(x, y, \eta)= & \left(1+x \partial_{x} B_{1,1}\left(0, y_{1}, y^{\prime}\right)\right) \eta_{1}^{2} \\
& +\sum_{j=1}^{d-1}\left(x \partial_{x} B_{1, j}(0, y)+O\left(x^{2}\right)\right) \eta_{1} \eta_{j}+\sum_{j, k=2}^{d-1} B_{j, k}(x, y) \eta_{j} \eta_{k} .
\end{aligned}
$$

Assumption 1.2 on the domain $\Omega$ is equivalent to saying that there exists a point $\left(0, y_{0}, \xi_{0}, \eta_{0}\right)$ on $T^{*} \Omega$ where the boundary is microlocally strictly convex, i.e. there exists a bicharacteristic passing through this point that intersects $\partial \Omega$ tangentially having exactly second order contact with the boundary and remaining in the complement of $\partial \bar{\Omega}$. If $p \in C^{\infty}\left(T^{*} X \backslash o\right)$ (where we write $o$ for the zero section) denotes the principal symbol of the wave operator $\partial_{t}^{2}-\Delta_{g}$, this last condition translates into

$$
\begin{gathered}
\tau^{2}=R\left(0, y_{0}, \eta_{0}\right), \quad\{p, x\}=\frac{\partial p}{\partial \xi}=2 \xi_{0}=0, \\
\{\{p, x\}, p\}=\left\{\frac{\partial p}{\partial \xi}, p\right\}=2 \partial_{x} R\left(0, y_{0}, \eta_{0}\right)>0,
\end{gathered}
$$

where $\left\{f_{1}, f_{2}\right\}$ denotes the Poisson bracket

$$
\left\{f_{1}, f_{2}\right\}=\frac{\partial f_{1}}{\partial \vartheta} \frac{\partial f_{2}}{\partial \rho}-\frac{\partial f_{1}}{\partial \rho} \frac{\partial f_{2}}{\partial \vartheta} .
$$

Denote the gliding point (in $T^{*} \Omega \times \mathbb{R}$ ) provided by Assumption 1.2 by

$$
\left(\rho_{0}, \vartheta_{0}\right)=\left(0, y_{0}, 0,0, \eta_{0}, \tau_{0}=-\sqrt{R\left(0, y_{0}, \eta_{0}\right)}\right) .
$$

We start the proof of Theorem 1.4 by reducing the problem to the two-dimensional case. Consider the following assumption: 
Assumption 2.1. Let $(\tilde{\Omega}, \tilde{g})$ be a smooth manifold of dimension 2 with $C^{\infty}$ boundary and Riemannian metric $\tilde{g}$. Assume that in local coordinates $\tilde{\Omega}=\{(x, \tilde{y}) \mid x>0, \tilde{y} \in \mathbb{R}\}$ and that the Laplace-Beltrami operator associated to $\tilde{g}$ is given by

$$
\partial_{x}^{2}+(1+x b(\tilde{y})) \partial_{\tilde{y}}^{2},
$$

where $b(\tilde{y})$ is a smooth function. Suppose in addition that there exists a bicharacteristic which intersects the boundary tangentially at $\left(0, \tilde{y}_{0}, \tilde{\xi}_{0}, \tilde{\eta}_{0}\right) \in T^{*} \tilde{\Omega}$ having exactly second order contact with the boundary and which remains in the complement of $\overline{\tilde{\Omega}}$. This is equivalent to saying that at $\left(0, \tilde{y}_{0}, \tilde{\xi}_{0}, \tilde{\eta}_{0}\right)$,

$$
\tilde{\xi}_{0}=0, \quad 2 b\left(\tilde{y}_{0}\right)>0 .
$$

We suppose in addition (without loss of generality, since we can always rescale the normal variable $x$ ) that $b\left(\tilde{y}_{0}\right)=1$.

Theorem 2.2. Let $(\tilde{\Omega}, \tilde{g})$ satisfy Assumption 2.1. Then there exists $T=T(\tilde{\Omega}) \in(0, \infty)$ and for every $\epsilon>0$ small enough there exist sequences $\tilde{V}_{h, j, \epsilon}, j \in\{0,1\}$, and approximate solutions $\tilde{V}_{h, \epsilon}$ to the wave equation on $\tilde{\Omega}$ with Dirichlet boundary conditions

$$
\left\{\begin{array}{l}
\partial_{t}^{2} V-\partial_{x}^{2} V-(1+x b(\tilde{y})) \partial_{\tilde{y}}^{2} V=0 \quad \text { on } \tilde{\Omega} \times \mathbb{R}, \\
\left.V\right|_{t=0}=\tilde{V}_{h, 0, \epsilon},\left.\quad \partial_{t} V\right|_{t=0}=\tilde{V}_{h, 1, \epsilon}, \\
\left.V\right|_{\partial \tilde{\Omega} \times[0, T]}=0,
\end{array}\right.
$$

which satisfy the following conditions:

(i) First, $\tilde{V}_{h, \epsilon}$ is an approximate solution to (2.4) in the sense that

$$
\partial_{t}^{2} \tilde{V}_{h, \epsilon}-\partial_{x}^{2} \tilde{V}_{h, \epsilon}-(1+x b(\tilde{y})) \partial_{\tilde{y}}^{2} \tilde{V}_{h, \epsilon}=O_{L^{2}(\tilde{\Omega})}(1 / h), \quad\left\|\tilde{V}_{h, \epsilon}\right\|_{L^{2}(\tilde{\Omega})} \leq 1 .
$$

(ii) Secondly, $\tilde{V}_{h, \epsilon}$ can be written as a sum

$$
\tilde{V}_{h, \epsilon}(x, \tilde{y}, t)=\sum_{n=0}^{N} v_{h, \epsilon}^{n}(x, \tilde{y}, t),
$$

where $1 \leq N \simeq h^{-(1-\epsilon) / 4}$ and where the functions $v_{h, \epsilon}^{n}(x, \tilde{y}, t)$ satisfy the following conditions:

- for $4<r<\infty$ :

$$
\left\{\begin{array}{l}
\left\|v_{h, \epsilon}^{n}(\cdot, t)\right\|_{L^{r}(\tilde{\Omega})} \geq C h^{-\frac{3}{2}\left(\frac{1}{2}-\frac{1}{r}\right)-\frac{1}{6}\left(\frac{1}{4}-\frac{1}{r}\right)+2 \epsilon}, \\
\sup \left(\left\|v_{h, \epsilon}^{n}(\cdot, t)\right\|_{L^{2}(\tilde{\Omega})}+h\left\|\partial_{t} v_{h, \epsilon}^{n}(\cdot, t)\right\|_{L^{2}(\tilde{\Omega})}\right) \leq 1,
\end{array}\right.
$$

where the constant $C=C(T)>0$ is independent of $h, \epsilon$ and $n$;

- $v_{h, \epsilon}^{n}(x, \tilde{y}, t)$ are essentially supported for the time variable $t$ in almost disjoint intervals of time $I_{n}$ satisfying $\left|I_{0}\right| \simeq\left|I_{n}\right| \simeq h^{(1-\epsilon) / 4}$ for all $n \in\{0, \ldots, N\}$, and also supported for the tangential variable $\tilde{y}$ in almost disjoint intervals; 
- $\tilde{V}_{h, \epsilon}$ are supported for the normal variable $0 \leq x \lesssim h^{(1-\epsilon) / 2}$ (where the implied constant depends only on $\tilde{\Omega}$ ) and localized at spatial frequency $1 / h$ in the tangential variable $\tilde{y}$. Moreover, uniformly in $\epsilon>0$,

$$
\sup _{\epsilon>0}\left\|\tilde{V}_{h, \epsilon}\right\|_{L^{2}(\tilde{\Omega})} \lesssim 1, \quad \sup _{\epsilon>0}\left\|\partial_{\tilde{y}} \tilde{V}_{h, \epsilon}\right\|_{L^{2}(\tilde{\Omega})} \lesssim \frac{1}{h}, \quad \sup _{\epsilon>0}\left\|\partial_{\tilde{y}}^{2} \tilde{V}_{h, \epsilon}\right\|_{L^{2}(\tilde{\Omega})} \lesssim \frac{1}{h^{2}} .
$$

In the rest of this section we show how Theorem 2.2 implies Theorem 1.4. Assume we have proved Theorem 2.2. Let $(\Omega, g)$ be a Riemannian manifold of dimension $d>2$ satisfying our assumptions in Theorem 1.4 and let $\left(0, y_{0}, \xi_{0}, \eta_{0}\right) \in T^{*} \Omega$ be a point satisfying (2.2), (2.3). From (2.1) it follows that local coordinates can be chosen such that $y_{0}=0 \in \mathbb{R}^{d-1}, \eta_{0}=(1,0, \ldots, 0) \in \mathbb{R}^{d-1}$ and such that the Laplace-Beltrami operator $\Delta_{g}$ is given by

$$
\Delta_{g}=\partial_{x}^{2}+\sum_{j, k=1}^{d-1} B_{j, k}(x, y) \partial_{j} \partial_{k},
$$

where for $x$ small enough

$$
B_{1,1}(x, y)=1+x \partial_{x} B_{1,1}(0, y)+O\left(x^{2}\right), \quad \partial_{x} B_{1,1}(0, y)>0
$$

and for $j \in\{2, \ldots, d-1\}$ we have $B_{1, j}(0, y)=0$. By rescaling the normal variable $x$, we can assume without loss of generality that $\partial_{x} B_{1,1}(0,0)=1$.

We can now define $\tilde{\Omega}$, locally in a neighborhood of $\left(x=0, y_{1}=0, \xi=0, \eta_{1}=0\right)$, to be the two-dimensional half-space $\tilde{\Omega}:=\left\{\left(x, y_{1}\right) \mid x>0, y_{1} \in \mathbb{R}\right\}$ equipped with the metric

$$
\tilde{g}\left(x, y_{1}, \xi, \eta_{1}\right):=\xi^{2}+\left(1+x b\left(y_{1}\right)\right) \eta_{1}^{2}, \quad b\left(y_{1}\right):=\partial_{x} B_{1,1}\left(0, y_{1}, 0\right) .
$$

Recall that we have assumed $b(0)=1$. Applying Theorem 2.2 near $\left(0, y_{1}=0,0, \eta_{1}=1\right)$ $\epsilon T^{*} \tilde{\Omega}$ we obtain, for $\epsilon>0$ small enough, sequences $\tilde{V}_{h, \epsilon, j}, j \in\{0,1\}$, such that the solution $\tilde{V}_{h, \epsilon}$ to (2.4) satisfies (2.5)-(2.7). Let $\chi \in C_{0}^{\infty}\left(\mathbb{R}^{d-2}\right)$ be a cut-off function supported in the coordinate chart such that $\chi=1$ in a neighborhood of $0 \in \mathbb{R}^{d-2}$, and for $j \in\{0,1\}$ set

$$
V_{h, \epsilon, j}\left(x, y_{1}, y^{\prime}\right):=h^{-(d-2) / 4} \tilde{V}_{h, \epsilon / 3, j}\left(x, y_{1}\right) e^{-\frac{\left|y^{\prime}\right|^{2}}{2 h}} \chi\left(y^{\prime}\right) .
$$

Proposition 2.3. Let $\Delta_{g}$ be given by (2.8). Then the solution $V_{h, \epsilon}$ to the wave equation (1.5) with Dirichlet boundary conditions and with initial data $\left(V_{h, \epsilon, 0}, V_{h, \epsilon, 1}\right)$ defined in (2.9) satisfies (1.6), (1.7).

Remark 2.4. Notice that Proposition 2.3 immediately implies Theorem 1.4. 
Proof. We proceed by contradiction. Let $(q, r)$ be a sharp wave admissible pair in dimension $d \in\{2,3,4\}$ with $r>4$ and set

$$
\beta(r, d)=\frac{d+1}{2}\left(\frac{1}{2}-\frac{1}{r}\right)+\frac{1}{6}\left(\frac{1}{4}-\frac{1}{r}\right) .
$$

We suppose to the contrary that the operator

$$
\sin \left(t \sqrt{-\Delta_{g}}\right): L^{2}(\Omega) \rightarrow L^{q}\left([0, T], L^{r}(\Omega)\right)
$$

is bounded by $h^{-\beta(r, d)+2 \epsilon}$, where $T=T(\tilde{\Omega})$ is given by Theorem 2.2 . Let $\tilde{V}_{h, \epsilon / 3}$ be the approximate solution to (2.4) with initial data $\left(\tilde{V}_{h, \epsilon / 3, j}\right)_{j=0,1}$ satisfying all the conditions in Theorem 2.2. For $t \in[0, T]$ we define

$$
W_{h, \epsilon}(x, y, t):=h^{-(d-2) / 4} \tilde{V}_{h, \epsilon / 3}\left(x, y_{1}, t\right) e^{-\frac{\left|y^{\prime}\right|^{2}}{2 h}} \chi\left(y^{\prime}\right) .
$$

Lemma 2.5. There exists a constant $c(T)>0$ independent of $h$ such that

$$
\begin{aligned}
& \left\|W_{h, \epsilon}\right\|_{L^{q}\left([0, T], L^{r}(\Omega)\right)} \geq c(T) h^{-\beta(r, d)+2 \epsilon / 3}, \\
& \left\|\left.W_{h, \epsilon}\right|_{t=0}\right\|_{L^{2}(\Omega)}+h\left\|\left.\partial_{t} W_{h, \epsilon}\right|_{t=0}\right\|_{L^{2}(\Omega)} \lesssim 1 .
\end{aligned}
$$

Proof. Indeed, using the special form of $\tilde{V}_{h, \epsilon}$ provided by Theorem 2.2 we can estimate

$$
\begin{aligned}
\left\|W_{h, \epsilon}\right\|_{L^{q}\left([0, T], L^{r}(\Omega)\right)}^{q} & =\int_{0}^{T}\left\|W_{h, \epsilon}\right\|_{L^{r}(\Omega)}^{q} d t \\
& =\left(\int_{0}^{T}\left\|\sum_{n=0}^{N} v_{h, \epsilon / 3}^{n}\right\|_{L^{r}(\tilde{\Omega})}^{q} d t\right) \times\left\|h^{-(d-2) / 4} e^{-\frac{\left|y^{\prime}\right|^{2}}{2 h}} \chi\left(y^{\prime}\right)\right\|_{L^{r}\left(\mathbb{R}^{d-2}\right)}^{q} \\
& \geq c h^{-\frac{q(d-2)}{2}\left(\frac{1}{2}-\frac{1}{r}\right)} \sum_{k \leq N} \int_{I_{k}}\left\|\sum_{n=0}^{N} v_{h, \epsilon / 3}^{n}\right\|_{L^{r}(\tilde{\Omega})}^{q} d t+O\left(h^{\infty}\right) \\
& \simeq c h^{-\frac{q(d-2)}{2}\left(\frac{1}{2}-\frac{1}{r}\right)} \sum_{k \leq N}\left|I_{k}\right|\left\|v_{h, \epsilon / 3}^{0}\right\|_{L^{r}(\tilde{\Omega})}^{q}+O\left(h^{\infty}\right) \\
& \simeq c T h^{-\frac{q(d-2)}{2}\left(\frac{1}{2}-\frac{1}{r}\right)}\left\|v_{h, \epsilon / 3}^{0}\right\|_{L^{r}(\tilde{\Omega})}^{q}+O\left(h^{\infty}\right) \\
& \geq c T h^{(-\beta(r, d)+2 \epsilon / 3) q} .
\end{aligned}
$$

We used here the fact that each $v_{h, \epsilon / 3}^{n}$ provided by Theorem 2.2 is essentially supported in time in an interval $I_{n}$ of size $1 / N$ and that $\left(I_{n}\right)_{n \in\{0, \ldots, N\}}$ are almost disjoint. Take $c(T)=(c T)^{1 / q}$, where $c$ is the bound from below of the integral in the $d-2$ tangential variables.

To estimate the $L^{2}(\Omega)$ norm we use again the fact that $v_{h, \epsilon}^{n}$ and its time derivative have disjoint essential supports in the tangential variable $y_{1}$. For $W_{h, \epsilon}(\cdot, 0)$ we have, for instance,

$$
\left\|\left.W_{h, \epsilon}\right|_{t=0}\right\|_{L^{2}(\Omega)}=\left\|\tilde{V}_{h, \epsilon / 3,0}\right\|_{L^{2}\left(x, y_{1}\right)}\left\|h^{-(d-2) / 4} e^{-\frac{\left|y^{\prime}\right|^{2}}{2 h}} \chi\left(y^{\prime}\right)\right\|_{L^{2}\left(\mathbb{R}^{d-2}\right)} \lesssim 1 .
$$


Let $V_{h, \epsilon}$ be the solution to (1.5) with data $\left(V_{h, \epsilon, j}\right)_{j=0,1}$ defined in (2.9) and write

$$
V_{h, \epsilon}=W_{h, \epsilon}+w_{h, \epsilon, \mathrm{err}}
$$

If we denote $\Delta_{\tilde{g}}=\partial_{x}^{2}+\left(1+x b\left(y_{1}\right)\right) \partial_{y_{1}}^{2}, \square_{\tilde{g}}=\partial_{t}^{2}-\Delta_{\tilde{g}}$, then $W_{h, \epsilon}$ solves

$$
\left\{\begin{array}{l}
\square_{\tilde{g}} W_{h, \epsilon}=\square_{\tilde{g}} \tilde{V}_{h, \epsilon / 3} h^{-(d-2) / 4} e^{-\frac{\left|y^{\prime}\right|^{2}}{2 h}} \chi\left(y^{\prime}\right), \\
\left.W_{h, \epsilon}\right|_{t=0}=V_{h, \epsilon, 0},\left.\quad \partial_{t} W_{h, \epsilon}\right|_{t=0}=V_{h, \epsilon, 1}, \\
\left.W_{h, \epsilon}\right|_{\partial \Omega \times[0, T]}=0 .
\end{array}\right.
$$

Since $V_{h, \epsilon}$ is a solution to (1.5), $w_{h, \epsilon, \text { err }}$ must satisfy

$$
\left\{\begin{aligned}
& \square_{g} w_{h, \epsilon, \mathrm{err}}=-\square_{\tilde{g}} \tilde{V}_{h, \epsilon / 3} h^{-(d-2) / 4} e^{-\frac{\left|y^{\prime}\right|^{2}}{2 h}} \chi\left(y^{\prime}\right)-\left(1+x b\left(y_{1}\right)\right) \partial_{y_{1}}^{2} W_{h, \epsilon} \\
&+\sum_{j, k=1}^{d-1} B_{j, k}(x, y) \partial_{y_{j}, y_{k}}^{2} W_{h, \epsilon}, \\
&\left.w_{h, \epsilon, \operatorname{err}}\right|_{t=0}=0,\left.\quad \partial_{t} w_{h, \epsilon, \mathrm{err}}\right|_{t=0}=0, \\
&\left.w_{h, \epsilon, \operatorname{err}}\right|_{\partial \Omega \times[0, T]}=0,
\end{aligned}\right.
$$

where we set $\square_{g}:=\partial_{t}^{2}-\Delta_{g}$ and we used that

$$
\Delta_{g}-\Delta_{\tilde{g}}=-\left(1+x b\left(y_{1}\right)\right) \partial_{y_{1}}^{2}+\sum_{j, k=1}^{d-1} B_{j, k}(x, y) \partial_{y_{j}, y_{k}}^{2}
$$

Lemma 2.6. For $t \in[0, T]$ the solution $w_{h, \epsilon, \mathrm{err}}$ to the wave equation (2.12) satisfies

$$
\begin{aligned}
\left\|\left(\partial_{t}^{2}-\Delta_{g}\right) w_{h, \epsilon, \operatorname{err}}(\cdot, t)\right\|_{L^{2}(\Omega)} & \lesssim h^{-2(1-(1-\epsilon / 3) / 2)}\left\|w_{h, \epsilon, \operatorname{err}}\right\|_{L^{2}(\Omega)} \simeq h^{-1-\epsilon / 3}, \\
\left\|\left(\partial_{t}^{2}-\Delta_{g}\right) w_{h, \epsilon, \operatorname{err}}(\cdot, t)\right\|_{\dot{H}^{-1}(\Omega)} & \lesssim h^{-\epsilon / 3}\left\|w_{h, \epsilon, \operatorname{err}}\right\|_{L^{2}(\Omega)} \simeq h^{-\epsilon / 3},
\end{aligned}
$$

where the estimates hold uniformly in $t \in[0, T]$ with constants independent of $\epsilon$ and of $h$. Moreover,

$$
\left\|w_{h, \epsilon, \operatorname{err}}\right\|_{L^{q}\left([0, T], L^{r}(\Omega)\right)} \leq C h^{-\beta(r, d)+2 \epsilon-\epsilon / 3},
$$

where $C=C(T)>0$ is independent of $\epsilon$.

Proof. We start with (2.15). Assume we have already proved (2.14). The Duhamel formula for $w_{h, \epsilon, \text { err reads }}$

$$
w_{h, \epsilon, \mathrm{err}}(x, y, t)=\int_{0}^{t} \frac{\sin \left((t-s) \sqrt{-\Delta_{g}}\right)}{\sqrt{-\Delta_{g}}}\left(\left(\partial_{t}^{2}-\Delta_{g}\right) w_{h, \epsilon, \mathrm{err}}(x, y, s)\right) d s
$$


Using the Minkowski inequality together with (2.13) we find

$$
\begin{aligned}
\left\|w_{h, \epsilon, \mathrm{err}}(\cdot, t)\right\|_{L^{r}(\Omega)} & =\left\|\int_{0}^{t} \frac{\sin \left((t-s) \sqrt{-\Delta_{g}}\right)}{\sqrt{-\Delta_{g}}}\left(\left(\partial_{t}^{2}-\Delta_{g}\right) w_{h, \epsilon, \mathrm{err}}(\cdot, s)\right) d s\right\|_{L^{r}(\Omega)} \\
& \lesssim \int_{0}^{t}\left\|\frac{\sin \left((t-s) \sqrt{-\Delta_{g}}\right)}{\sqrt{-\Delta_{g}}}\left(\left(\partial_{t}^{2}-\Delta_{g}\right) w_{h, \epsilon, \mathrm{err}}(\cdot, s)\right)\right\|_{L^{r}(\Omega)} d s \\
& \lesssim h^{-\beta(r, d)+2 \epsilon}\left\|\left(\sqrt{-\Delta_{g}}\right)^{-1}\left(\partial_{t}^{2}-\Delta_{g}\right) w_{h, \epsilon, \operatorname{err}}\right\|_{L^{1}\left([0, T], L^{2}(\Omega)\right)} \\
& \simeq h^{-\beta(r, d)+2 \epsilon}\left\|\left(\partial_{t}^{2}-\Delta_{g}\right) w_{h, \epsilon, \operatorname{err}}\right\|_{L^{1}\left([0, T], \dot{H}^{-1}(\Omega)\right)} \\
& \lesssim h^{-\beta(r, d)+2 \epsilon-\epsilon / 3},
\end{aligned}
$$

where in the third line we used that the wave operator $\sin \left(t \sqrt{-\Delta_{g}}\right)$ was supposed to be bounded by $h^{-\beta(r, d)+2 \epsilon}$ and where in the last line we used (2.13). The implied constant in the last inequality depends only on $T$ and the estimates hold uniformly with respect to $t \in[0, T]$.

To prove (2.13) and (2.14), we use the special form of $\Delta_{g}$ and the fact that $\tilde{V}_{h, \epsilon / 3}\left(x, y_{1}, t\right)$ (and thereforee $\left.V_{h, \epsilon}\right)$ is supported in $0 \leq x \lesssim h^{(1-\epsilon / 3) / 2}$. The inhomogeneous part of the equation in (2.12) reads

$$
\square \tilde{V}_{h, \epsilon / 3} h^{-(d-2) / 4} e^{-\frac{\left|y^{\prime}\right|^{2}}{2 h}} \chi\left(y^{\prime}\right)+\left(1+x b\left(y_{1}\right)\right) \partial_{y_{1}}^{2} W_{h, \epsilon}-\sum_{j, k=1}^{d-1} B_{j, k}(x, y) \partial_{y_{j}, y_{k}}^{2} W_{h, \epsilon} .
$$

The $L^{2}(\Omega)$ norm of $\square \tilde{V}_{h, \epsilon / 3} h^{-(d-2) / 4} e^{-\left|y^{\prime}\right|^{2} /(2 h)} \chi\left(y^{\prime}\right)$ is estimated using the last condition in Theorem 2.2 and its contribution to the norm of the nonlinear term of (2.12) is $O_{L^{2}(\Omega)}(1 / h)$. We estimate the second term in (2.16) as follows:

$$
\begin{aligned}
-\left(1+x b\left(y_{1}\right)\right) \partial_{y_{1}}^{2} W_{h, \epsilon}+B_{1,1}(x, y) \partial_{y_{1}, y_{1}}^{2} W_{h, \epsilon} \\
=h^{-(d-2) / 4} e^{-\frac{\left|y^{\prime}\right|^{2}}{2 h}} \chi\left(y^{\prime}\right) \partial_{y_{1}}^{2} \tilde{V}_{h, \epsilon / 3}\left(B_{1,1}(x, y)-1-b\left(y_{1}\right)\right) .
\end{aligned}
$$

The last term in (2.16) splits into two sums, corresponding to $k=1$,

$$
\begin{aligned}
& \sum_{j=1}^{d-1} B_{1, j}(x, y) \partial_{y_{1}, y_{j}}^{2} W_{h, \epsilon} \\
& \quad=-h^{-(d-2) / 4} e^{-\frac{\left|y^{\prime}\right|^{2}}{2 h}} \frac{1}{h} \partial_{y_{1}} \tilde{V}_{h, \epsilon / 3} \sum_{j=2}^{d-1} B_{1, j}(x, y)\left(y_{j} \chi\left(y^{\prime}\right)+h \partial_{y_{j}} \chi\left(y^{\prime}\right)\right),
\end{aligned}
$$

and $k \in\{2, \ldots, d-1\}$, respectively,

$$
\begin{aligned}
& \sum_{j, k=2}^{d-1} B_{j, k}(x, y) \partial_{y_{j}, y_{k}}^{2} W_{h, \epsilon}=h^{-(d-2) / 4} e^{-\frac{\left|y^{\prime}\right|^{2}}{2 h}} \frac{1}{h^{2}} B_{j, k}(x, y) \tilde{V}_{h, \epsilon / 3} \\
& \quad \times \sum_{j, k=2}^{d-1}\left(y_{j} y_{k} \chi\left(y^{\prime}\right)-h\left(y_{j} \partial_{y_{k}} \chi\left(y^{\prime}\right)+y_{k} \partial_{y_{j}} \chi\left(y^{\prime}\right)+\delta_{j=k}\right)+h^{2} \partial_{y_{j}, y_{k}}^{2} \chi\left(y^{\prime}\right)\right) .
\end{aligned}
$$


If $\left|y^{\prime}\right| \geq h^{\left(1-\epsilon^{\prime}\right) / 2}$ for some $\epsilon^{\prime}>0$, then $e^{-\left|y^{\prime}\right|^{2} /(2 h)} \leq C_{M} h^{M}$ for all $M \geq 0$, thus taking $\epsilon^{\prime}=\epsilon / 3$ we can estimate the $L^{2}(\Omega)$ norm of (2.17) and (2.18) as follows:

$$
\begin{aligned}
&\left\|-\left(1+x b\left(y_{1}\right)\right) \partial_{y_{1}}^{2} W_{h, \epsilon}+\sum_{j, k=1}^{d-1} B_{j, k}(x, y) \partial_{y_{j}} \partial_{y_{k}} W_{h, \epsilon}\right\|_{L^{2}(\Omega)} \\
& \lesssim h^{-2+(1-\epsilon / 3)}\left\|\tilde{V}_{h, \epsilon / 3}\right\|_{L^{2}(\tilde{\Omega})} \lesssim h^{-1-\epsilon / 3}
\end{aligned}
$$

where we used that

$$
\sup _{\epsilon>0}\left\|\tilde{V}_{h, \epsilon / 3}\right\|_{L^{2}(\tilde{\Omega})} \lesssim 1, \quad \sup _{\epsilon>0}\left\|\partial_{y_{1}} \tilde{V}_{h, \epsilon / 3}\right\|_{L^{2}(\tilde{\Omega})} \lesssim \frac{1}{h}, \quad \sup _{\epsilon>0}\left\|\partial_{y_{1}}^{2} \tilde{V}_{h, \epsilon / 3}\right\|_{L^{2}(\tilde{\Omega})} \lesssim \frac{1}{h^{2}}
$$

In the same way we obtain the bounds

$$
\begin{aligned}
\|-(1+ & \left.x b\left(y_{1}\right)\right) \partial_{y_{1}}^{2} W_{h, \epsilon}+\sum_{j, k=1}^{d-1} B_{j, k}(x, y) \partial_{y_{j}, y_{k}}^{2} W_{h, \epsilon} \|_{\dot{H}^{-1}(\Omega)} \\
& \lesssim h\left\|-\left(1+x b\left(y_{1}\right)\right) \partial_{y_{1}}^{2} W_{h, \epsilon}+\sum_{j, k=1}^{d-1} B_{j, k}(x, y) \partial_{y_{j}, y_{k}}^{2} W_{h, \epsilon}\right\|_{L^{2}(\Omega)} \lesssim h^{-\epsilon / 3} .
\end{aligned}
$$

For the last inequality we used the following lemma (see [10, Prop. 5.4] for the proof):

Lemma 2.7. Let $f(x, y): \Omega \rightarrow \mathbb{R}$ be localized at frequency $1 / h$ in the $y \in \mathbb{R}^{d-1}$ variable, i.e. such that there exists $\psi \in C_{0}^{\infty}\left(\mathbb{R}^{d-1} \backslash\{0\}\right)$ with $\psi\left(h D_{y}\right) f=f$. Then there exists a constant $C>0$ independent of $h$ such that

$$
\|f\|_{\dot{H}^{-1}(\Omega)} \leq C h\|f\|_{L^{2}(\Omega)} .
$$

End of proof of Proposition 2.3. Recall that we have assumed that the operator

$$
\sin \left(t \sqrt{-\Delta_{g}}\right): L^{2}(\Omega) \rightarrow L^{q}\left([0, T], L^{r}(\Omega)\right)
$$

is bounded by $h^{-\beta(r, d)+2 \epsilon}$. This assumption implies

$$
\begin{aligned}
\left\|V_{h, \epsilon}\right\|_{L^{q}\left([0, T], L^{r}(\Omega)\right)} & \leq C h^{-\beta(r, d)+2 \epsilon}\left(\left\|V_{h, \epsilon, 0}\right\|_{L^{2}(\Omega)}+\left\|V_{h, \epsilon, 1}\right\|_{\dot{H}^{-1}(\Omega)}\right) \\
& \leq \tilde{C} h^{-\beta(r, d)+2 \epsilon},
\end{aligned}
$$

where $C, \tilde{C}>0$ are independent of $h$. Now (2.19) together with (2.10) gives

$$
\begin{aligned}
h^{-\beta(r, d)+2 \epsilon / 3} & \lesssim\left\|W_{h, \epsilon}\right\|_{L^{q}\left([0, T], L^{r}(\Omega)\right)} \\
& \lesssim\left(\left\|V_{h, \epsilon}\right\|_{L^{q}\left([0, T], L^{r}(\Omega)\right)}+\left\|w_{h, \epsilon, \operatorname{err}}\right\|_{L^{q}\left([0, T], L^{r}(\Omega)\right)}\right) .
\end{aligned}
$$

The last estimate together with (2.15) and (2.19) gives a contradiction, since it would imply

$$
h^{-\beta(r, d)+2 \epsilon / 3} \lesssim h^{-\beta(r, d)+2 \epsilon}+h^{-\beta(r, d)+2 \epsilon-\epsilon / 3},
$$

which obviously cannot be true. The proof is complete. 


\section{Proof of Theorem 1.4}

In order to finish the proof of Theorem 1.4 it remains to prove Theorem 2.2. Let $(\Omega, g)$ be a Riemannian manifold of dimension $d=2$ with $C^{\infty}$ boundary $\partial \Omega$. Suppose that local coordinates can be chosen near $(x=0, y=0)$ such that in a neighborhood of this point $\Omega$ is given by

$$
\Omega=\{(x, y) \mid x>0, y \in \mathbb{R}\},
$$

and the Laplace-Beltrami operator $\Delta_{g}$ associated to the metric $g$ is given by

$$
\Delta_{g}=\partial_{x}^{2}+(1+x b(y)) \partial_{y}^{2},
$$

where $b$ is a smooth function with $b(0)=0$. Let $Y>0$ be such that for $y \in[0, Y]$ we have $\left|b^{1 / 3}(y)-1\right| \leq \frac{1}{10}$. We proceed with the proof of Theorem 2.2 , where we write $(\Omega, g)$ instead of $(\tilde{\Omega}, \tilde{g})$ and where $\Delta_{g}$ is given by (3.1).

Remark 3.1. In what follows we fix $\epsilon>0$ small enough and we do not mention anymore the dependence on $\epsilon$ of the solution to the wave equation (2.4) we are going to construct.

Before starting the proof of Theorem 2.2 we briefly recall some definitions we shall use in the rest of the paper (for details see [9] or [27], for example).

Set $X=\Omega \times \mathbb{R}_{t}$, let $\square_{g}=\partial_{t}^{2}-\Delta_{g}$ denote the wave operator on $X$ and let $p \in$ $C^{\infty}\left(T^{*} X \backslash o\right)$ be the principal symbol of $\square_{g}$, which is homogeneous of degree 2 in $T^{*} X \backslash o$,

$$
p(x, y, t, \xi, \eta, \tau)=\xi^{2}+(1+x b(y)) \eta^{2}-\tau^{2} .
$$

The characteristic set $P:=\operatorname{Char}(p) \subset T^{*} X \backslash o$ of $\square_{g}$ is defined to be $p^{-1}(\{0\})$.

Let us consider the Dirichlet problem for $\square_{g}$ :

$$
\square_{g} u=0,\left.\quad u\right|_{\partial X}=0 .
$$

The statement of the propagation of singularities of solutions to (3.3) has two main ingredients: locating singularities of a distribution, as captured by the wave front set, and describing the curves along which they propagate, namely the bicharacteristics. Both of these are closely related to an appropriate notion of "phase space", in which both the wave front set and the bicharateristics are located. On manifolds without boundary, this phase space is the standard cotangent bundle $T^{*} X$. In the presence of boundaries it is the $b$ cotangent bundle, ${ }^{b} T^{*} X$. There is a natural noninjective "inclusion" $\pi: T^{*} X \rightarrow{ }^{b} T^{*} X$. We define the elliptic, glancing and hyperbolic sets in $T^{*} \partial X$ as follows:

$$
\begin{aligned}
\mathcal{E} & =\left\{q \in \pi\left(T^{*} X\right) \backslash o \mid \pi^{-1}(q) \cap \operatorname{Char}(p)=\emptyset\right\}, \\
\mathcal{G} & =\left\{q \in \pi\left(T^{*} X\right) \backslash o \mid \operatorname{Card}\left(\pi^{-1}(q) \cap \operatorname{Char}(p)\right)=1\right\}, \\
\mathcal{H} & =\left\{q \in \pi\left(T^{*} X\right) \backslash o \mid \operatorname{Card}\left(\pi^{-1}(q) \cap \operatorname{Char}(p)\right) \geq 2\right\},
\end{aligned}
$$

with Card denoting the cardinality of a set; each of these is a conic subset of $\pi\left(T^{*} X\right) \backslash o$. Note that in $T^{*} \stackrel{\circ}{X}, \pi$ is the identity map, so every point $q \in T^{*} \stackrel{\circ}{X}$ is either elliptic or glancing, depending on whether $q \notin \operatorname{Char}(p)$ or $q \in \operatorname{Char}(p)$. 
The canonical local coordinates on $T^{*} X$ will be denoted $(x, y, t, \xi, \eta, \tau)$, so 1 -forms are $\alpha=\xi d x+\eta d y+\tau d t$. Let $(\rho, \vartheta)=(x, y, t, \xi, \eta, \tau)$ on $T^{*} X$ near $\pi^{-1}(q), q \in$ $T^{*} \partial X$, with corresponding coordinates $(y, t, \eta, \tau)$ on a neighborhood $\mathcal{U}$ of $q$ in $T^{*} \partial X$. Consequently,

$$
\begin{aligned}
& \mathcal{E} \cap \mathcal{U}=\left\{(y, t, \eta, \tau) \mid \tau^{2}<\eta^{2}\right\}, \\
& \mathcal{G} \cap \mathcal{U}=\left\{(y, t, \eta, \tau) \mid \tau^{2}=\eta^{2}\right\}, \\
& \mathcal{H} \cap \mathcal{U}=\left\{(y, t, \eta, \tau) \mid \tau^{2}>\eta^{2}\right\} .
\end{aligned}
$$

Let $\rho=\rho(s)=(x, y, t)(s), \vartheta=\vartheta(s)=(\xi, \eta, \tau)(s)$ be a bicharacteristic of $p(\rho, \vartheta)$, i.e. such that $(\rho, \vartheta)$ satisfies

$$
\frac{d \rho}{d s}=\frac{\partial p}{\partial \vartheta}, \quad \frac{d \vartheta}{d s}=-\frac{\partial p}{\partial \rho}, \quad p(\rho(0), \vartheta(0))=0 .
$$

We say that $\left.(\rho(s), \vartheta(s))\right|_{s=0}$ on the boundary $\partial X$ is a gliding point if

$$
x(\rho(0))=0, \quad \frac{d}{d s} x(\rho(0))=0, \quad \frac{d^{2}}{d s^{2}} x(\rho(0))<0 .
$$

This is equivalent to saying that $(\rho, \vartheta) \in T^{*} X \backslash o$ is a gliding point if

$$
p(\rho, \vartheta)=0, \quad\{p, x\}_{\left.\right|_{(\rho, \vartheta)}}=0, \quad\{\{p, x\}, p\}_{\left.\right|_{(\rho, \vartheta)}}>0 .
$$

Our assumption on the domain $\Omega$ in Theorem 2.2 near the point $(x=0, y=0)$ is equivalent to saying that there exists a bicharacteristic intersecting tangentially the boundary at $(0,0)$ and having second order contact with the boundary at this point. From (3.4) this last condition translates into the following: there exists $\left(\xi_{0}, \eta_{0}, \tau_{0}\right)$ such that

$$
\begin{gathered}
\tau_{0}^{2}=(1+x b(y)) \eta_{\left.\right|_{\left(0,0, \xi_{0}, \eta_{0}\right)} ^{2}}^{2}, \quad\{p, x\}_{\left.\right|_{\left(0,0, \xi_{0}, \eta_{0}\right)}}=\left.\frac{\partial p}{\partial \xi}\right|_{\left.\right|_{\left(0,0, \xi_{0}, \eta_{0}\right)}}=2 \xi_{0}=0, \\
\{\{p, x\}, p\}_{\left.\right|_{\left(0,0, \xi_{0}, \eta_{0}\right)}}=\left\{\frac{\partial p}{\partial \xi}, p\right\}_{\left.\right|_{\left(0,0, \xi_{0}, \eta_{0}\right)}}=2 b(0) \eta_{0}^{2}>0 .
\end{gathered}
$$

Since $b(0)=1$ we must have $\xi_{0}=0$ and $\eta_{0} \neq 0$. Suppose without loss of generality that $\eta_{0}=1$. Let $\left(\rho_{0}, \vartheta_{0}\right)=\left(x=0, y=0, t=0, \xi_{0}=0, \eta_{0}=1, \tau_{0}=-1\right)$ and denote the gliding point (in $T^{*} \partial X$ ) by

$$
\pi\left(\rho_{0}, \vartheta_{0}\right)=\left(y=0, t=0, \eta_{0}, \tau_{0}=-\eta_{0}\right)=(0,0,1,-1) \in \mathcal{G} .
$$

We define the semi-classical wave front set $\mathrm{WF}_{h}(u)$ of a distribution $u$ on $\mathbb{R}^{3}$ to be the complement of the set of points $(\rho=(x, y, t), \zeta=(\xi, \eta, \tau)) \in \mathbb{R}^{3} \times\left(\mathbb{R}^{3} \backslash 0\right)$ for which there exists a symbol $a(\rho, \zeta) \in \mathcal{S}\left(\mathbb{R}^{6}\right)$ such that $a(\rho, \zeta) \neq 0$ and for all integers $m \geq 0$,

$$
\left\|a\left(\rho, h D_{\rho}\right) u\right\|_{L^{2}} \leq c_{m} h^{m} .
$$




\subsection{Choice of an approximate solution}

We look for an approximate solution to the equation (2.4) of the form

$$
u_{h}(x, y, t)=\int e^{\frac{i}{h}\left(\theta+\zeta \xi+\xi^{3} / 3\right)} g_{h} d \xi d \eta d \tau,
$$

where the phase functions $\theta(x, y, t, \eta, \tau), \zeta(x, y, \eta, \tau)$ are real valued and homogeneous in $(\eta, \tau)$ of degree 1 and $2 / 3$, respectively, and where $g_{h}$ is a symbol to be determined in the next sections. In order for $u_{h}$ to solve (2.4), the functions $\theta, \zeta$ must solve an eikonal equation that we derive in what follows. We denote by $\langle\cdot, \cdot\rangle$ the symmetric bilinear form obtained by polarization of the second order homogeneous principal symbol $p$ of the wave operator $\square_{g}$,

$$
\langle d a, d b\rangle=\partial_{x} a \partial_{x} b+(1+x b(y)) \partial_{y} a \partial_{y} b-\partial_{t} a \partial_{t} b .
$$

Applying the wave operator $h^{2} \square_{g}$ to $u_{h}$, the main contribution becomes

$$
\begin{array}{r}
\left(\partial_{x} \theta+\xi \partial_{x} \zeta\right)^{2}+(1+x b(y))\left(\partial_{y} \theta+\xi \partial_{y} \zeta\right)^{2}-\left(\partial_{t} \theta+\xi \partial_{t} \zeta\right)^{2} \\
=\langle d \theta, d \theta\rangle-2 \xi\langle d \theta, d \zeta\rangle+\xi^{2}\langle d \zeta, d \zeta\rangle
\end{array}
$$

In order to eliminate this term after integrations by parts in $\xi$ we require the right hand side of (3.7) to be a nontrivial multiple of $\partial_{\xi} \Phi$, where we set

$$
\Phi=\theta+\zeta \xi+\xi^{3} / 3 .
$$

This is equivalent to determining $\theta, \zeta$ solutions to

$$
\left\{\begin{array}{l}
\langle d \theta, d \theta\rangle-\zeta\langle d \zeta, d \zeta\rangle=0 \\
\langle d \theta, d \zeta\rangle=0
\end{array}\right.
$$

The system (3.8) is a nonlinear system of partial differential equations, which is elliptic where $\zeta>0$ (shadow region), hyperbolic where $\zeta<0$ (illuminated region) and parabolic where $\zeta=0$ (caustic curve or surface). It is crucial that there is a solution of the form

$$
\phi^{ \pm}=\theta \mp \frac{2}{3}(-\zeta)^{3 / 2}
$$

with $\theta, \zeta$ smooth. Solutions with such singularities arise from solving the initial value problem for (3.8) off an initial surface which does not have the usual transversality condition, corresponding to the fact that there are bicharacteristics tangent to the boundary.

3.1.1. Geometric reduction. Let $X=\Omega \times \mathbb{R}$ as before. Let $p$ and $q$ be functions on $T^{*} X$ with independent differentials at a point $(\rho, \vartheta) \in T^{*} X \backslash o$. We denote by $P$ and $Q$ the hypersurfaces defined by $p$ and $q$, respectively. 
Definition 3.2. We say that the hypersurfaces $P, Q$ in the symplectic manifold $T^{*} X$ are glancing surfaces at $(\rho, \vartheta)$ if

1. $\{p, q\}((\rho, \vartheta))=0$,

2. $\{p,\{p, q\}\}((\rho, \vartheta)) \neq 0$ and $\{q,\{q, p\}\}((\rho, \vartheta)) \neq 0$.

In our case we take $q$ to be the defining function of the boundary $\partial \Omega$, therefore $q=x$, and $p$ the symbol of the wave operator $\square_{g}$ defined in (3.2). Precisely,

$$
Q=\{q(x, y, t, \xi, \eta, \tau)=x=0\}, \quad P=\left\{p=\xi^{2}+(1+x b(y)) \eta^{2}-\tau^{2}=0\right\},
$$

which are glancing at $\left(\rho_{0}, \vartheta_{0}\right)$ defined in (3.5). The nondegeneracy conditions in Definition 3.2 hold at a point $(\rho, \vartheta)$ with $\{p, q\}=0$ if and only if $\partial \Omega$ is strictly convex at $(\rho, \vartheta)$.

Remark 3.3. A model case of a pair of glancing surfaces is given by

$$
Q_{F}=\left\{q_{F}(x, y, \xi, \eta, \tau)=x=0\right\}, \quad P_{F}=\left\{p_{F}=\xi^{2}+(1+x) \eta^{2}-\tau^{2}=0\right\},
$$

which have a second order intersection at the point

$$
\left(\bar{\rho}_{0}, \bar{\vartheta}_{0}\right):=\left(0, y_{0}=0, t_{0}=0,0, \eta_{0}=1, \tau_{0}=-1\right) \in T^{*} X_{F} \backslash o .
$$

This model case was studied in [10]. There is a deep geometrical reason underlying the similarity of the general gliding ray parametrix for (3.9) and the one for the model example (3.10), which will facilitate solution to the eikonal equation.

Theorem 3.4. Let $P$ and $Q$ be two hypersurfaces in $T^{*} X \backslash$ o satisfying the glancing conditions in Definition 3.2 at $\left(\rho_{0}, \vartheta_{0}\right) \in P \cap Q \subset T^{*} X \backslash$ o. Then there exist real functions $\theta$ and $\zeta$ which are $C^{\infty}$ in a conic neighborhood $\mathcal{U}$ of $\left(\rho_{0}, 1,-1\right) \in X \times \mathbb{R}^{2}$, are homogeneous of degree one and two-thirds, respectively, and have the following properties:

(i) $\zeta_{0}:=\left.\zeta\right|_{x=0}=-\left(\tau^{2}-\eta^{2}\right) \eta^{-4 / 3}$ and $\left.\partial_{x} \zeta\right|_{\partial X}>0$ on $\mathcal{U} \cap \partial X \times \mathbb{R}^{2}$,

(ii) $d_{y, t}\left(\partial_{\eta} \theta, \partial_{\tau} \theta\right)$ are linearly independent on $\mathcal{U}$,

(iii) the system (3.8) holds in $\zeta \leq 0$; moreover, the phases $\theta$ and $\zeta$ also satisfy (3.8) to infinite order at $x=0$.

Moreover, $\zeta$ is a defining function for the fold set denoted $\Sigma$. By translation invariance in time, $\zeta$ is independent of $t$ while the phase function $\theta$ is linear in the time variable.

Remark 3.5. Theorem 3.4 has been proved independently by Melrose in [18] and by Eskin in [6, Thm. 1] for the canonical glancing surfaces

$$
Q_{\text {can }}=\{x=0\}, \quad P_{\text {can }}=\left\{\xi^{2}+x \eta^{2}-\tau \eta=0\right\},
$$

near the glancing point ( $x=0, y, t, \xi=0, \eta=1, \tau=0$ ). It is not difficult to see that the phase functions $\theta$ and $\zeta$ can be chosen to satisfy the conditions stated above and therefore we leave the details of the proof of Theorem 3.4 to the reader. 
Remark 3.6. Notice that if $P$ and $Q$ are the hypersurfaces in the symplectic space $T^{*} X \backslash o$ defined in (3.9) and glancing at $\left(\rho_{0}, \vartheta_{0}\right) \in T^{*} X \backslash o$, then there exists a canonical transformation

$$
\chi: \Gamma \subset T^{*} X_{F} \backslash o \rightarrow T^{*} X \backslash o,
$$

defined in a conic neighborhood $\Gamma$ of $\left(\bar{\rho}_{0}, \bar{\vartheta}_{0}\right)$ and taking $\left(\bar{\rho}_{0}, \bar{\vartheta}_{0}\right)$ to $\left(\rho_{0}, \vartheta_{0}\right)$ and the model pair $P_{F}$ and $Q_{F}$ to $P$ and $Q$. The fact that $\chi$, which is symplectic, maps $Q_{F}$ onto $Q$ means that it defines a local canonical transformation from the quotient space of $Q_{F}$, modulo its Hamilton fibration, to the corresponding quotient space of $Q$, which is naturally identified as the cotangent space of the hypersurface

$$
Q / \mathbb{R} H_{q} \simeq T^{*} \partial X
$$

Now, as we just said, on $Q$ (and similarly on $Q_{F}$ ) the symplectic form gives a Hamilton foliation. Let it determine an equivalence relation $\sim$. Then $Q \cap P / \sim$ has the structure of a symplectic manifold with boundary and it is naturally isomorphic to the closure of the "hyperbolic" set in $T^{*} \partial X$, the region over which real rays pass, and similarly for $Q_{F} \cap P_{F} / \sim$. Therefore, the restriction of $\chi$ to $T^{*} \partial X_{F}$, denoted $\chi_{\partial}$, is also a canonical transformation from a neighborhood $\gamma \subset T^{*} \partial X_{F} \backslash o$ of $\pi\left(\bar{\rho}_{0}, \bar{\vartheta}_{0}\right)$ to a neighborhood of $\pi\left(\rho_{0}, \vartheta_{0}\right) \in T^{*} \partial X \backslash o$,

$$
\begin{gathered}
\chi_{\partial}: \gamma \rightarrow T^{*} \partial X \backslash o, \quad \gamma \subset T^{*} \partial X_{F} \backslash o, \\
\gamma=\left\{(y, t, \eta, \tau) \in T^{*} \partial X_{F} \mid \exists \xi,(0, y, t, \xi, \eta, \tau) \in \Gamma\right\}
\end{gathered}
$$

defined in the hyperbolic region by

$$
\chi_{\partial}^{-1}:\left(y, t, d_{y} \theta_{0}, d_{t} \theta_{0}\right) \mapsto\left(d_{\eta} \theta_{0}, d_{\tau} \theta_{0}, \eta, \tau\right), \quad \chi_{\partial}^{-1}\left(\pi\left(\rho_{0}, \vartheta_{0}\right)\right)=\pi\left(\bar{\rho}_{0}, \bar{\vartheta}_{0}\right),
$$

where $\theta_{0}:=\left.\theta\right|_{\partial X}$ is the restriction to $\partial X$ of the phase $\theta$ introduced in Theorem 3.4.

The map $\chi_{\partial}$ has the property that near $\pi\left(\bar{\rho}_{0}, \bar{\vartheta}_{0}\right)$ it conjugates the billiard ball map $\delta^{ \pm} \subset\left(T^{*} \partial X \backslash o\right) \times\left(T^{*} \partial X \backslash o\right)$ to the normal form $\delta_{F}^{ \pm}$introduced in (3.14) below. An interpolating Hamiltonian for the billiard ball maps $\delta^{ \pm}$is $\zeta_{0}$ and $\delta^{ \pm}(y, t, \eta, \tau)=$ $\exp \left( \pm \frac{4}{3} H_{\left(-\zeta_{0}\right)^{3 / 2}}\right)$.

Remark 3.7. In Friedlander's model domain where $\Omega_{F}:=\left\{(x, y) \in \mathbb{R}_{+} \times \mathbb{R}\right\}$ and $\Delta_{F}:=\partial_{x}^{2}+(1+x) \partial_{y}^{2}$, which we have dealt with in [10], the equation (3.8) has a solution of the form

$$
\phi_{F}^{ \pm}=\theta_{F} \mp \frac{2}{3}\left(-\zeta_{F}\right)^{3 / 2},
$$

where

$$
\theta_{F}(x, y, t, \eta, \tau)=y \eta+t \tau, \quad \zeta_{F}(x, y, \eta, \tau)=\left(x-\frac{\tau^{2}-\eta^{2}}{\eta^{2}}\right) \eta^{2 / 3},
$$

as can be seen by direct computation. This solution serves very much as a guide to the general construction as we shall see in the next sections. 


\subsection{A model operator}

Since the heart of the matter is well illustrated by Friedlander's example, we start by recalling the main steps of the construction in [10] where we proved Theorem 1.4 in the case of a two-dimensional, strictly convex domain $\Omega_{F}=\left\{(x, y) \in \mathbb{R}_{+} \times \mathbb{R}\right\}$ with Laplace operator given by

$$
\Delta_{F}=\partial_{x}^{2}+(1+x) \partial_{y}^{2}
$$

Let $X_{F}=\Omega_{F} \times \mathbb{R}$ and let $p_{F} \in C^{\infty}\left(T^{*} X_{F} \backslash o\right)$ denote the homogeneous symbol of the model wave operator $\square_{F}, p_{F}(x, y, t, \xi, \eta, \tau)=\xi^{2}+(1+x) \eta^{2}-\tau^{2}$. Consider the wave equation

$$
\left\{\begin{array}{l}
\partial_{t}^{2} v-\partial_{x}^{2} v-(1+x) \partial_{y}^{2} v=0 \\
\left.v\right|_{\partial \Omega_{F} \times[0, T]}=0
\end{array}\right.
$$

In [10] we have constructed a parametrix for (3.12) of the form

$$
U_{F, h}(x, y, t)=\sum_{n=0}^{N} u_{F, h}^{n}(x, y, t)
$$

where $u_{F, h}^{n}$ are approximate solutions to (3.12) of the form

$$
u_{F, h}^{n}(x, y, t)=\int e^{\frac{i}{h} \eta\left(y-t(1+a)^{1 / 2}+\xi(x-a)+\frac{\xi^{3}}{3}+\frac{4}{3} n a^{3 / 2}\right)} g_{F}^{n}(t, \xi, \eta, h) d \xi d \eta
$$

where $g_{F}^{n}$ are smooth functions compactly supported for $\eta$ near 1 and where the relation between the amplitudes in the sum is dictated by the billiard ball maps. In this case, due to the presence of the translations in $(y, t)$, the billiard ball maps have specific formulas

$$
\delta_{F}^{ \pm}(y, t, \eta, \tau)=\left(y \pm 4\left(\frac{\tau^{2}}{\eta^{2}}-1\right)^{1 / 2} \pm \frac{8}{3}\left(\frac{\tau^{2}}{\eta^{2}}-1\right)^{3 / 2}, t \mp 4\left(\frac{\tau^{2}}{\eta^{2}}-1\right)^{1 / 2} \frac{\tau}{\eta}, \eta, \tau\right) .
$$

The associated Lagrangian sets are defined by

$$
\Lambda_{F, n}:=\left\{\xi^{2}+(x-a)=0, y-t(1+a)^{1 / 2}+\xi(x-a)+\frac{\xi^{3}}{3}+\frac{4}{3} n a^{3 / 2}=0\right\} \subset T^{*} X_{F} \backslash o .
$$

Remark 3.8. Notice that, on the boundary, $\Lambda_{F, n}$ is the graph of the canonical transformation $\left(\delta_{F}^{ \pm}\right)^{n}$ given by the formula

$$
\left(\delta_{F}^{ \pm}\right)^{n}(y, t, \eta, \tau)=\left(y \pm 4 n\left(\frac{\tau^{2}}{\eta^{2}}-1\right)^{1 / 2} \pm \frac{8}{3} n\left(\frac{\tau^{2}}{\eta^{2}}-1\right)^{3 / 2}, t \mp 4 n\left(\frac{\tau^{2}}{\eta^{2}}-1\right)^{1 / 2} \frac{\tau}{\eta}, \eta, \tau\right) .
$$


We introduce the fold set $\Sigma_{F}=\{\xi=0\}$ as the set of singular points of the canonical projection $\Lambda_{F, n} \rightarrow X_{F}$ and we define the caustic set to be the image of $\Sigma_{F}$ through this canonical projection, hence the set $\left\{x-\left(\tau^{2}-\eta^{2}\right) / \eta^{2}=0\right\}=\left\{\zeta_{F}(x, y, \eta, \tau)=0\right\}$. Near the caustic set $\left\{\zeta_{F}=0\right\}$ each solution $u_{F, h}^{n}$ "lives" essentially on a cusp defined by

$$
y-t(1+a)^{1 / 2}+\frac{4}{3} n a^{3 / 2}= \pm(a-x)^{3 / 2} .
$$

The key observation is that, if the parameter $a$ is small enough, depending on the frequency, each such cusp type solution provides a loss in the Strichartz estimates.

3.2.1. Construction of an approximate solution in the model case. In this section we recall the construction from [10] of the symbols $g_{F}^{n}$ of the cusp type parametrices $u_{F, h}^{n}$ in (3.13) for the model wave operator $\square_{F}$. We take $a$ of the form $a \simeq h^{\alpha}$ for some $\alpha$ to be chosen later as large as possible in the interval $(0,2 / 3)$; notice that the case $a \simeq h^{2 / 3}$ corresponds to the whispering gallery modes dealt with in [10], while the case $a \simeq 1$ describes a wave transverse to the boundary.

Definition 3.9. Let $\lambda \geq 1$. For a given compact $K \subset \mathbb{R}$ we define the space $\mathcal{S}_{K}(\lambda)$ to consist of all functions $\varrho(z, \lambda) \in C^{\infty}(\mathbb{R})$ which satisfy

1. $\sup _{z \in \mathbb{R}, \lambda \geq 1}\left|\partial_{z}^{\alpha} \varrho(z, \lambda)\right| \leq C_{\alpha}$, where $C_{\alpha}$ are constants independent of $\lambda$,

2. if $\psi(z) \in C_{0}^{\infty}$ is a smooth function equal to 1 in a neighborhood of $K$ and $0 \leq \psi \leq 1$ then $(1-\psi) \varrho \in O_{\mathcal{S}(\mathbb{R})}\left(\lambda^{-\infty}\right)$.

Here $\mathcal{S}(\mathbb{R})$ denotes the Schwartz space of rapidly decreasing functions.

We define a new parameter $\lambda=a^{3 / 2} / h \gg 1$ and for some small $0<c_{0} \leq 3 / 8$, we set $K_{0}=\left[-c_{0}, c_{0}\right]$. We take $\varrho(\cdot, \lambda) \in \mathcal{S}_{K_{0}}(\lambda)$ and set

$$
g_{F}^{0}(t, \xi, \eta, h)=\varrho\left(\frac{t+2(1+a)^{1 / 2} \xi}{2(1+a)^{1 / 2} a^{1 / 2}}, \lambda\right) \Psi(\eta),
$$

where $\Psi \in C_{0}^{\infty}(\mathbb{R} \backslash\{0\})$ is supported in a small neighborhood of 1 and $0 \leq \Psi(\eta) \leq 1$. The boundary condition will help us determine the symbols $g_{F}^{n}$ for every $0 \leq n \leq N$.

Proposition 3.10 ([10, Proposition 3.3, see also Lemma 3.2]). On the boundary $u_{F, h}^{0}$ can be (modulo $\mathrm{O}_{L^{2}}\left(\lambda^{-\infty}\right)$ ) as a sum of two trace operators,

$$
u_{F, h}^{0}(0, y, t)=\sum_{ \pm} \operatorname{Tr}_{ \pm}\left(u_{F, h}^{0}\right)(y, t),
$$

where

$$
\begin{aligned}
& \operatorname{Tr}_{ \pm}\left(u_{F, h}^{0}\right)(y, t):=h^{1 / 3} \int e^{\frac{i}{h}\left(y \eta-t(1+a)^{1 / 2} \eta \mp \frac{2}{3} a^{3 / 2} \eta\right)} \Psi(\eta)(\eta \lambda)^{-1 / 6} \\
& \times I_{ \pm}(\varrho(\cdot, \lambda))_{\eta}\left(\frac{t}{2(1+a)^{1 / 2} a^{1 / 2}}, \lambda\right) d \eta
\end{aligned}
$$


with $I_{ \pm}(\varrho(\cdot, \lambda))_{\eta}(z, \lambda)$ given by

$$
I_{ \pm}(\varrho(\cdot, \lambda))_{\eta}(z, \lambda)=\frac{\eta \lambda}{2 \pi} \int e^{i \eta \lambda\left(w\left(z-z^{\prime}\right) \mp \frac{2}{3}\left((1-w)^{3 / 2}-1\right)\right)} \kappa(w) a_{ \pm}(w, \eta \lambda) \varrho\left(z^{\prime}, \lambda\right) d w .
$$

Here $\kappa$ is a smooth function supported for $w$ in a small neighborhood of 0 such that $0 \leq \kappa \leq 1$ and $\kappa(w)=1$ for $w$ close to 0 . In the integral (3.17), $a_{ \pm}$are given by the asymptotic expansions of the Airy function $\operatorname{Ai}\left(-(\lambda \eta)^{2 / 3}(1-w)\right)$,

$$
a_{ \pm}(w, \eta, \lambda) \simeq e^{ \pm i \pi / 2-i \pi / 4}(1-w)^{-1 / 4} \sum_{j \geq 0} a_{ \pm, j}(-1)^{-j / 2}(1-w)^{-3 j / 2}(\eta \lambda)^{-j} .
$$

Precisely, we used the decomposition $\operatorname{Ai}(z)=A_{+}(z)+A_{-}(z)$, where $A_{ \pm}(z)=$ $\mathrm{Ai}\left(e^{\mp 2 \pi i / 3} z\right)$. In particular, using the properties of the Airy functions $A_{ \pm}$it follows that the symbols $k(w) a_{ \pm}(w, \eta \lambda)$ are elliptic at $w=0$ (see [10, Appendix]).

Proposition 3.11 ([10, Lemma 3.4]). Let $p \in \mathbb{Z}$ and $K_{p}=\left[-c_{0}+p, c_{0}+p\right]$. Then for $\eta$ belonging to the support of $\Psi$ we have

$$
I_{ \pm, \eta}: \mathcal{S}_{K_{p}}(\lambda) \rightarrow \mathcal{S}_{K_{p \mp 1}}(\lambda) .
$$

Proposition 3.12. Let $\eta$ belong to the support of $\Psi$ and let $J_{ \pm, \eta}$ be the operators defined for some $\tilde{\lambda} \geq 1$ and $\breve{\varrho} \in \mathcal{S}_{K \mp 1}(\tilde{\lambda})$ by the formula

$$
J_{ \pm}(\breve{\varrho}(\cdot, \tilde{\lambda}))_{\eta}\left(z^{\prime}, \lambda\right):=\frac{\eta \lambda}{2 \pi} \int e^{i \eta \lambda\left(\left(z^{\prime}-z\right) w \pm \frac{2}{3}\left((1-w)^{3 / 2}-1\right)\right)} b_{ \pm}(w, \eta \lambda) \breve{\varrho}(z, \tilde{\lambda}) d z d w
$$

where $b_{ \pm}(w, \eta \lambda)=\frac{k(w)}{a_{ \pm}(w, \eta \lambda)}$ are asymptotic expansions in $(\eta \lambda)^{-1}$. Then

$$
\begin{aligned}
& \breve{\varrho}(\cdot, \tilde{\lambda})=I_{ \pm}\left(J_{ \pm}(\breve{\varrho}(\cdot, \tilde{\lambda}))_{\eta}(\cdot, \lambda)\right)_{\eta}(\cdot, \lambda)+O_{\mathcal{S}(\mathbb{R})}\left(\lambda^{-\infty}\right)+O_{\mathcal{S}(\mathbb{R})}\left(\tilde{\lambda}^{-\infty}\right) \\
& \varrho(\cdot, \tilde{\lambda})=J_{ \pm}\left(I_{ \pm}(\varrho(\cdot, \tilde{\lambda}))_{\eta}(\cdot, \lambda)\right)_{\eta}(\cdot, \lambda)+O_{\mathcal{S}(\mathbb{R})}\left(\lambda^{-\infty}\right)+O_{\mathcal{S}(\mathbb{R})}\left(\tilde{\lambda}^{-\infty}\right) .
\end{aligned}
$$

The construction of the operators $J_{ \pm, \eta}$ is detailed in [10, Section 3.3.1].

Proposition 3.13 ([10, Proposition 3.6]). Let $N \lesssim \lambda h^{\epsilon}$ for some small $\epsilon>0$ and let $1 \leq n \leq N$. Let $T_{k}$ denote the translation operator which to a given function $\varrho(z)$ associates $\varrho(z+k)$. Then for $\eta \in \operatorname{supp}(\Psi)$ we have

$$
\left(T_{1} \circ J_{+}(\cdot)_{\eta} \circ I_{-}(\cdot)_{\eta} \circ T_{1}\right)^{\circ n}: \mathcal{S}_{K_{0}}(\lambda) \rightarrow \mathcal{S}_{K_{0}}(\lambda / n) \quad \text { uniformly in } n .
$$

Notice that since $\lambda / n \geq h^{-\epsilon} \gg 1$, then $O_{\mathcal{S}(\mathbb{R})}\left(\lambda^{-\infty}\right)=O_{\mathcal{S}(\mathbb{R})}\left((\lambda / n)^{-\infty}\right)=O_{\mathcal{S}(\mathbb{R})}\left(h^{\infty}\right)$.

Moreover, the operator defined above can be written as a convolution

$$
\left(T_{1} \circ J_{+}(\cdot)_{\eta} \circ I_{-}(\cdot)_{\eta} \circ T_{1}\right)^{\circ n}(\varrho)=\left(F_{\eta \lambda}\right)^{* n} * \varrho, *
$$

where

$$
\left(F_{\eta \lambda}\right)^{* n}(z)=\frac{\eta \lambda}{2 \pi} \int e^{i \eta \lambda\left(w z+n\left(2 w+\frac{4}{3}\left((1-w)^{3 / 2}-1\right)\right)\right.}\left(\kappa(w) a_{+}(w, \eta \lambda) b_{-}(w, \eta \lambda)\right)^{n} d w .
$$


Definition 3.14. Let $\varrho(\cdot, \lambda) \in \mathcal{S}_{K_{0}}(\lambda)$ and $\eta \in \operatorname{supp}(\Psi)$. For $1 \leq n \leq N, N \lesssim \lambda h^{\epsilon}$ set

$$
\varrho^{n}(z, \eta, \lambda):=(-1)^{n}\left(T_{1} \circ J_{+}(\cdot)_{\eta} \circ I_{-}(\cdot)_{\eta} \circ T_{1}\right)^{n}(\varrho(\cdot, \lambda))(z), \quad \varrho^{0}(z, \eta, \lambda)=\varrho(z, \lambda) .
$$

Remark 3.15. From Proposition 3.13 it follows that $\varrho^{n}(z, \eta, \lambda) \in \mathcal{S}_{K_{0}}(\lambda / n)$.

Definition 3.16. For $0 \leq n \leq N$ with $N \lesssim \lambda h^{\epsilon}$ define

$$
g_{F}^{n}(t, \xi, \eta, h):=\varrho^{n}\left(\frac{t+2(1+a)^{1 / 2} \xi}{2(1+a)^{1 / 2} a^{1 / 2}}-2 n, \eta, \lambda\right) \Psi(\eta)
$$

Proposition 3.17. For all $0 \leq n \leq N-1$ we have

$$
\operatorname{Tr}_{-}\left(u_{F, h}^{n}\right)(y, t)+\operatorname{Tr}_{+}\left(u_{F, h}^{n+1}\right)(y, t)=O_{L^{2}}\left(\lambda^{-\infty}\right) .
$$

Proposition 3.18. If $0 \leq n \leq N, u_{F, h}^{n}(\cdot, y, t)$ is essentially supported for $y$ and $t$ in the interval

$$
I_{n}\left(c_{0}\right):=2 a^{1 / 2}(1+a)^{1 / 2} \times\left[2 n-\left(1+c_{0}\right), 2 n+\left(1+c_{0}\right)\right],
$$

i.e. for $y$ or $t$ outside any neighborhood of $I_{n}\left(c_{0}\right)$ the contribution of $u_{F, h}^{n}$ is $O_{L^{2}}\left(h^{\infty}\right)$.

\subsection{Construction of an approximate solution in the general case}

In this section we construct an approximate solution to (2.4) satisfying the conditions of Theorem 2.2. It will be essentially based on the model construction and Theorem 3.4.

Inspired from [10], we construct superposition solutions $u_{h}^{n}$ to (2.4) of the form

$$
u_{h}^{n}(x, y, t)=\int e^{\frac{i}{h} \Phi^{n}(x, y, t, \xi, \eta, \tau)} g_{h}^{n} d \xi d \eta d \tau
$$

for some symbols $g_{h}^{n}$ to be suitably chosen and where the phase functions are given by

$$
\Phi^{n}(x, y, t, \xi, \eta, \tau):=\theta(x, y, t, \eta, \tau)+\eta^{1 / 3} \xi \zeta(x, y, \eta, \tau)+\eta \frac{\xi^{3}}{3}+\frac{4}{3} n\left(-\zeta_{0}\right)^{3 / 2}(\eta, \tau) .
$$

We determine the symbols $g_{h}^{n}$ in (3.19) so that $u_{h}^{n}$ is an approximate solution to (2.4) in a sense to be made precise. We start by defining their restriction to the boundary by requiring the Dirichlet condition to be fulfilled. We consider an operator $J$ defined by

$$
J(f)(y, t):=\frac{1}{(2 \pi h)^{2}} \int e^{\frac{i}{h} \theta_{0}(y, t, \eta, \tau)} d_{h}(y, \eta, \tau) \widehat{f}(\eta / h, \tau / h) d \eta d \tau,
$$

where $d_{h}(y, \eta, \tau)=d(y, \eta / h, \tau / h)$ for some elliptic symbol $d(y, \eta, \tau)$ of order 0 and type $(1,0)$, compactly supported in a conic neighborhood of the glancing point $\pi\left(\rho_{0}, \vartheta_{0}\right)$. Here $\theta_{0}$ denotes the restriction to the boundary of the phase $\theta$ introduced in Theorem 3.4.

The operator $J$ defines an elliptic FIO in a neighborhood of $\left(\pi\left(\bar{\rho}_{0}, \bar{\vartheta}_{0}\right), \pi\left(\rho_{0}, \vartheta_{0}\right)\right)$, with canonical relation $\chi_{\partial}$ given by the symplectomorphism generated by $\theta_{0}$ which satisfies $\chi_{\partial}\left(\pi\left(\bar{\rho}_{0}, \bar{\vartheta}_{0}\right)\right)=\pi\left(\rho_{0}, \vartheta_{0}\right)$ (see the remarks following Theorem 3.4).

In what follows we compute $J \circ \operatorname{Tr}_{ \pm}\left(u_{F, h}^{n}\right)$, where $0 \leq n \leq N$ for some $N$ to be determined later. We keep the notation of Section 3.2. 
Proposition 3.19. On the boundary, $J \circ \operatorname{Tr}_{ \pm}\left(u_{F, h}^{n}\right)$ reads

$$
\begin{aligned}
J \circ \operatorname{Tr}_{ \pm}\left(u_{F, h}^{n}\right)(y, t)=h^{1 / 3} & \int e^{\frac{i}{h}\left(\theta_{0}\left(y, t, \eta,-\eta(1+a)^{1 / 2}\right)+\frac{2}{3}(2 n \mp 1)\left(-\zeta_{0}\right)^{3 / 2}\left(\eta,-\eta(1+a)^{1 / 2}\right)\right)}(\lambda \eta)^{-1 / 6} \\
& \times I_{ \pm}\left(g_{h}^{n}(\cdot, y, \eta)\right)_{\eta}\left(\frac{\partial_{\tau} \theta_{0}\left(y, t, \eta,-\eta(1+a)^{1 / 2}\right)}{2(1+a)^{1 / 2} a^{1 / 2}}-2 n, \lambda\right) d \eta
\end{aligned}
$$

where

$$
g_{h}^{n}(z, y, \eta) \simeq \Psi(\eta)\left(\sum_{k \geq 0} h^{k / 2} a^{-k / 2} \mu_{k}(y, \eta, h) \partial_{z}^{k} \varrho^{n}(z, \eta, \lambda)\right) .
$$

Here $\mu_{k}(y, \eta, h)$ are symbols of order 0 and type $(1,0)$ independent of $n$. Moreover, if $\eta \in \operatorname{supp}(\Psi)$ and $1 \leq n \leq N \lesssim \lambda h^{\epsilon}$ for some small $\epsilon>0$ then $g_{h}^{n}(\cdot, y, \eta) \in \mathcal{S}_{K_{0}}(\lambda / n)$.

Proof. An explicit computation (using arguments from [10, Lemma 3.2]) gives

$$
\begin{aligned}
u_{F, h}^{n}(0, \bar{y}, \bar{t})=h^{1 / 3} \sum_{ \pm} & \int e^{\frac{i}{h}\left(\bar{y} \bar{\eta}-\bar{t}(1+a)^{1 / 2} \bar{\eta} \mp \frac{2}{3} a^{3 / 2} \bar{\eta}+\frac{4}{3} n a^{3 / 2} \bar{\eta}\right)} \Psi(\bar{\eta})(\bar{\eta} \lambda)^{-1 / 6} \\
& \times I_{ \pm}\left(\varrho^{n}(\cdot, \bar{\eta}, \lambda)\right)_{\bar{\eta}}\left(\frac{\bar{t}}{2(1+a)^{1 / 2} a^{1 / 2}}-2 n, \lambda\right) d \bar{\eta}
\end{aligned}
$$

where $I_{ \pm}\left(\varrho^{n}(\cdot, \bar{\eta}, \lambda)\right)_{\bar{\eta}}(z, \lambda)$ are defined in (3.17). The contributions corresponding to the \pm signs in the right hand side of (3.23) are denoted $\operatorname{Tr}_{ \pm}\left(u_{F, h}^{n}\right)(\bar{y}, \bar{t})$.

We can now proceed to compute $J \circ \operatorname{Tr}_{ \pm}\left(u_{F, h}^{n}\right)(\bar{y}, \bar{t})$ :

$$
\begin{aligned}
& J \circ \operatorname{Tr}_{ \pm}\left(u_{F, h}^{n}\right)(y, t)=\frac{h^{1 / 3}}{(2 \pi h)^{2}} \int e^{\frac{i}{h}\left(\theta_{0}(y, t, \eta, \tau)-\bar{y}(\eta-\bar{\eta})-\bar{t}\left(\tau+\bar{\eta}(1+a)^{1 / 2}\right) \mp \frac{2}{3} a^{3 / 2} \bar{\eta}+\frac{4}{3} n a^{3 / 2} \bar{\eta}\right)} \Psi(\bar{\eta}) \\
& \quad \times I_{ \pm}\left(\varrho^{n}(\cdot, \bar{\eta}, \lambda)\right)_{\bar{\eta}}\left(\frac{\bar{t}}{2(1+a)^{1 / 2} a^{1 / 2}}-2 n, \lambda\right)(\bar{\eta} \lambda)^{-1 / 6} d_{h}(y, \eta, \tau) d \bar{\eta} d \bar{y} d \bar{t} d \eta d \tau .
\end{aligned}
$$

Since the symbol is independent of $\bar{y}$, integration in $\bar{y}$ gives $\eta=\bar{\eta}$. Now we are in a situation where the stationary phase theorem can be applied in the variables $(\bar{t}, \tau)$. Consequently, $J \circ \operatorname{Tr}_{ \pm}\left(u_{F, h}^{n}\right)$ admits the asymptotic expansion

$$
\begin{gathered}
J \circ \operatorname{Tr}_{ \pm}\left(u_{F, h}^{n}\right)(y, t) \simeq h^{1 / 3} \int e^{\frac{i}{h}\left(\theta_{0}\left(y, t, \eta,-\eta(1+a)^{1 / 2}\right)+\frac{2}{3}(2 n \mp 1)\left(-\zeta_{0}\right)^{3 / 2}\left(\eta,-\eta(1+a)^{1 / 2}\right)\right)} \Psi(\eta) \\
\times\left[\sum_{k \geq 0} h^{k / 2} a^{-k / 2} \mu_{k}(y, \eta, h) \partial^{k} I_{ \pm}\left(\varrho^{n}(\cdot, \eta, \lambda)\right)_{\eta}\left(\frac{\partial_{\tau} \theta_{0}\left(y, t, \eta,-\eta(1+a)^{1 / 2}\right)}{2(1+a)^{1 / 2} a^{1 / 2}}-2 n, \lambda\right)\right] \\
\times(\eta \lambda)^{-1 / 6} d \eta,
\end{gathered}
$$

where we set

$$
\mu_{k}(y, \eta, h)=\left.i^{k} 2^{-k} h^{k / 2}(1+a)^{-k / 2} \partial^{k}\left(e^{\frac{i}{h} r(y, t, \eta, \tau)} d_{h}(y, \eta, \tau)\right)\right|_{\left\{\begin{array}{l}
\tau=-\eta(1+a)^{1 / 2} \\
\bar{t}=\partial_{\tau} \theta_{0}\left(y, t, \eta,-\eta(1+a)^{1 / 2}\right.
\end{array}\right.}
$$


The main contribution of $\mu_{2 v}$ is equal to $\left(\partial_{\tau}^{2} r\right)^{v} d_{h} e^{\frac{i}{h} r(\omega, \tau)}$ and that of $\mu_{2 v-1}$ is $h\left(\partial_{\tau}^{2} r\right)^{\nu} \partial_{\tau} d_{h} e^{\frac{i}{h} r(\omega, \tau)}$, all the other terms in the sum defining $\mu_{k}$ being positive powers of $h$. Since $d_{h}$ is a symbol of order 0 and type $(1,0)$, we deduce that $\mu_{k}$ is also a symbol of order 0 and type $(1,0)$.

Notice, moreover, that $I_{ \pm}\left(\varrho^{n}\right)_{\eta}$ is a convolution product and consequently $\partial^{k}\left(I_{ \pm}\left(\varrho^{n}\right)_{\eta}\right)=I_{ \pm}\left(\partial^{k} \varrho^{n}\right)_{\eta}$. Since from Proposition 3.13 and Definition 3.14 the symbols $\varrho^{n}(\cdot, \eta, \lambda)$ belong to $\mathcal{S}_{K_{0}}(\lambda / n)$, where $K_{0}=\left[-c_{0}, c_{0}\right]$, it follows that the sum

$$
\Psi(\eta)\left(\sum_{k \geq 0} h^{k / 2} a^{-k / 2} \mu_{k}(y, \eta, h) \partial^{k} \varrho^{n}(z, \eta, \lambda)\right)
$$

(denoted $g_{h}^{n}(z, y, \eta)$ in the statement of Proposition 3.19) also belongs to $\mathcal{S}_{K_{0}}(\lambda / n)$.

Lemma 3.20. Let $\Phi^{n}$ be defined by (3.20). Then the integral curves of the vector field $\left\langle 2 d \Phi^{n}, d \cdot\right\rangle-\eta^{-1 / 3}\langle d \zeta, d \zeta\rangle \partial_{\xi}$ are given by

$$
\eta^{-2 / 3} \zeta+\xi^{2}, \quad \partial_{\tau} \theta+\eta^{1 / 3} \xi \partial_{\tau} \zeta
$$

Now we can define $u_{h}^{n}$ everywhere as follows:

Definition 3.21. Let $g_{h}^{n}$ be the symbol defined in (3.22) and for $0 \leq n \leq N \lesssim \lambda h^{\epsilon}$ let

$$
\begin{aligned}
u_{h}^{n}(x, y, t) & :=\int e^{\frac{i}{h} \Phi^{n}\left(x, y, t, \xi, \eta,-\eta(1+a)^{1 / 2}\right)} \\
& \times g_{h}^{n}\left(\frac{\partial_{\tau} \theta+\eta^{1 / 3} \xi \partial_{\tau} \zeta}{2(1+a)^{1 / 2} a^{1 / 2}}\left(x, y, t, \eta,-\eta(1+a)^{1 / 2}\right)-2 n, y, \eta\right) d \xi d \eta .
\end{aligned}
$$

It remains to show that the restriction to $\partial \Omega$ of $u_{h}^{n}$ defined in (3.25) coincides with the sum of the two terms in (3.24). We leave the proof of Proposition 3.22 below to the reader.

Proposition 3.22. On the boundary $\partial \Omega$ we have, indeed,

$$
u_{h}^{n}(0, y, t)=\sum_{ \pm} J\left(\operatorname{Tr}_{ \pm}\left(u_{F, h}^{n}\right)\right)(y, t, h) .
$$

Moreover,

$$
J\left(\operatorname{Tr}_{-}\left(u_{F, h}^{n}\right)\right)(y, t)+J\left(\operatorname{Tr}_{+}\left(u_{F, h}^{n+1}\right)\right)(y, t)=O_{L^{2}}\left(h^{\infty}\right) .
$$

Applying the wave operator $\square_{g}$ to $u_{h}^{n}$ (defined by (3.25)) and using (3.8) yields

$$
\begin{aligned}
\square_{g} u_{h}^{n}(x, y, t) & =\int e^{\frac{i}{h} \Phi^{n}\left(x, y, t, \xi, \eta,-\eta(1+a)^{1 / 2}\right)} \\
& \times\left(\frac{i}{h}\left(\left\langle 2 d \Phi^{n}, d g_{h}^{n}\right\rangle-\eta^{-1 / 3}\langle d \zeta, d \zeta\rangle \partial_{\xi} g_{h}^{n}+\left(\square_{g} \Phi^{n}\right) g_{h}^{n}\right)+\square g_{h}^{n}\right) d \xi d \eta
\end{aligned}
$$

Using Lemma 3.20 and performing some elementary computations, we obtain:

Proposition 3.23. The following estimates hold uniformly for $0 \leq n \leq N \lesssim \lambda h^{\epsilon}$ :

$$
\left\|\square_{g} u_{h}^{n}(\cdot, t)\right\|_{L^{2}(\Omega)}=O\left(h^{-1}\right)\left\|u_{h}^{n}(\cdot, t)\right\|_{L^{2}(\Omega)} .
$$




\subsection{Main properties of the parametrix}

In this section we state the main properties of the parametrix

$$
U_{h}(x, y, t):=\sum_{n=0}^{N} u_{h}^{n}(x, y, t),
$$

where $u_{h}^{n}(x, y, t)$ are introduced in (3.25) and where $N \lesssim \lambda h^{\epsilon}$ for some $\epsilon>0$. Here $\epsilon$ is fixed at the beginning of Section 3 (see Remark 3.1). We first prove that each $u_{h}^{n}$ is essentially supported for $t$ in an interval of size $\simeq a^{1 / 2}$ and that $\left(u_{h}^{n}\right)_{n}$ have almost disjoint supports in time and in the tangential variable $y$.

In the first part of this section we choose $N$ so that for all $0 \leq n \leq N$, each cusp type solution $u_{h}^{n}$ preserves the same properties as $u_{h}^{0}$. We prove that this requires

$$
4 N a^{1 / 2} \lesssim Y
$$

Taking into account that we need to impose $N \lesssim \lambda h^{\epsilon}$ (since otherwise the construction in Section 3.2 may degenerate) and since we require the parameter $a$ to be as small as possible, we must have

$$
N \simeq \lambda h^{\epsilon}, \text { where } \lambda=a^{3 / 2} / h
$$

The last conditions yield $a \simeq \frac{1}{2} Y^{1 / 2} h^{(1-\epsilon) / 2}$. We state a useful property of the parametrix $u_{h}^{n}$ :

Proposition 3.24. Let $u_{h}^{n}$ be given by (3.25). Then the wave front set $\mathrm{WF}_{h}\left(u_{h}^{n}\right)$ of $u_{h}^{n}$ is contained in the Lagrangian set $\Lambda_{\Phi^{n}}$ defined by

$$
\begin{aligned}
\Lambda_{n}:=\left\{\left(x, y, t, \xi, \eta,-\eta(1+a)^{1 / 2}\right) \mid \zeta\left(x, y, \eta,-\eta(1+a)^{1 / 2}\right)+\eta^{2 / 3} \xi^{2}=0,\right. & \\
& \left.\left(\partial_{\eta} \theta-(1+a)^{1 / 2} \partial_{\tau} \theta+\xi \zeta\right)\left(x, y, t, 1,-(1+a)^{1 / 2}\right)+\frac{\xi^{3}}{3}+\frac{4}{3} n a^{3 / 2}=0\right\} .
\end{aligned}
$$

In other words, outside any neighborhood of $\Lambda_{n}$ the contribution of $u_{h}^{n}$ is $O_{L^{2}}\left(h^{\infty}\right)$.

Proposition 3.24 follows from integrations by parts either in the variable $\xi$, using the operator $L_{1}:=\frac{h}{i} \frac{1}{\xi^{2}+\eta^{-2 / 3} \zeta} \partial_{\xi}$, or in $\eta$, using $L_{2}:=\frac{h}{i} \frac{\partial_{\eta} \Phi^{n}}{\left|\partial_{\eta} \Phi^{n}\right|^{2}} \partial_{\eta}$. We need however to estimate the $\eta$-derivatives of $g_{h}^{n}$, and in order to do so we use the convolution type form of $\varrho^{n}$ which follows from Proposition 3.13.

3.4.1. Number of iterations and time interval. In what follows we choose the number $N$ of iterated cusp type solutions $u_{h}^{n}$. 
Proposition 3.25. Let $1 \leq N \leq C_{0} Y a^{-1 / 2}$ for some fixed $C_{0}>0$. If $C_{0}$ is chosen sufficiently small, then the operator $J$ introduced in (3.21) is elliptic near the set

$$
\left(\bigcup_{0 \leq n \leq N} I_{n}\left(c_{0}\right)\right) \times\left(\bigcup_{0 \leq n \leq N} I_{n}\left(c_{0}\right)\right) \times\left(1,-(1+a)^{1 / 2}\right)
$$

and $\chi_{\partial}$ is a diffeomorphism from a neighborhood of this set onto its image. We recall that $I_{n}\left(c_{0}\right)$ was introduced in (3.18) and $0<c_{0} \leq 3 / 8$ was fixed in Section 3.2.1.

Proof. We first deal with the last statement. We use the properties of $\chi_{\partial}$ defined in Section 3.1.1 together with the assumption on $b(y)$ in Theorem 2.2. We can explicitly compute the gradient $\nabla_{y, t}\left(\chi_{\partial}^{-1}\right)$ to be

$$
\left(\begin{array}{ll}
\partial_{y, \eta}^{2} \theta_{0}(y, t, \eta, \tau) & 0 \\
\partial_{y, \tau}^{2} \theta_{0}(y, t, \eta, \tau) & 1
\end{array}\right) .
$$

Using the construction of the phases $\theta_{0}$ and $\zeta_{0}$ we obtain

$$
\partial_{y, \eta}^{2} \theta_{0}(y, t, \eta, \tau)=b^{2 / 3}(y)+O\left(\zeta_{0}(\eta, \tau)\right)
$$

and also

$$
\partial_{y, \tau}^{2} \theta_{0}(y, t, \eta, \tau)=\left(1-\left(\frac{\tau}{\eta}\right)^{4 / 3} b^{2 / 3}(y)\right)+O\left(\zeta_{0}(\eta, \tau)\right) .
$$

Notice also that the right hand side in both (3.29) and (3.30) is independent of $t$ (which follows from the linearity in time of $\theta$ ). Since $\left|b^{1 / 3}(y)-1\right| \leq 1 / 10$ for $y \in[0, Y]$, if $\eta / \tau$ is close to 1 it follows that $\chi_{\partial}$ is a diffeomorphism from a small, fixed, conic neighborhood of $\pi\left(\bar{\varrho}_{0}, \bar{\vartheta}_{0}\right)$ into a small neighborhood of $\pi\left(\varrho_{0}, \vartheta_{0}\right)$.

Remark 3.26. In particular, the Jacobian of the restriction of $\chi_{\partial}^{-1}$ to the values $(\eta, \tau)=$ $\left(1,-(1+a)^{1 / 2}\right)$ equals $\operatorname{Jac}\left(\chi_{\partial}^{-1}\right)=\partial_{y, \eta}^{2} \theta_{0}\left(y, t, 1,-(1+a)^{1 / 2}\right)$ and is independent of $t$.

Now we proceed with the first part of Proposition 3.25. Recall that $\chi_{\partial}$ conjugates the billiard ball map $\delta^{ \pm}$to the normal form, $\chi_{\partial} \circ \delta_{F}^{ \pm}=\delta^{ \pm} \circ \chi_{\partial}$. Since we consider only positive time it is enough to work with $\delta^{+}, \delta_{F}^{+}$. For $n \geq 1$ and for $\pi(\bar{\varrho}, \bar{\vartheta})$ in a small, conic neighborhood of $\pi\left(\bar{\varrho}_{0}, \bar{\vartheta}_{0}\right)$ we have, writing $\pi(\varrho, \vartheta)=\chi_{\partial}(\pi(\bar{\varrho}, \bar{\vartheta}))$,

$$
\left(\delta^{+}\right)^{n}(\pi(\varrho, \vartheta))=\left(\delta^{+}\right)^{n}\left(\chi_{\partial}(\pi(\bar{\varrho}, \bar{\vartheta}))\right)=\chi_{\partial}\left(\left(\delta_{F}^{+}\right)^{n}(\pi(\bar{\varrho}, \bar{\vartheta}))\right),
$$

where $\left(\delta_{F}^{+}\right)^{n}$ is given by (3.15). We have assumed (without loss of generality) that $\pi\left(\varrho_{0}, \vartheta_{0}\right)=(0,0,1,-1)$. Modulo a translation we can also assume that $\pi\left(\bar{\varrho}_{0}, \bar{\vartheta}_{0}\right)=$ $(0,0,1,-1)$. We now take $\bar{\varrho}=\bar{\varrho}_{0}$ and $\bar{\vartheta}=\left(1,-(1+a)^{1 / 2}\right)$ in $(3.31)$. Therefore, for $a$ small, depending on $h, \pi(\bar{\varrho}, \bar{\vartheta})$ belongs to a conic neighborhood of $\pi\left(\bar{\varrho}_{0}, \bar{\vartheta}_{0}\right)$. Rewriting (3.31) at this point and using (3.15) yields

$$
\begin{aligned}
\left(\delta^{+}\right)^{n}(\pi(\varrho, \vartheta)) & =\chi_{\partial}\left(4 n a^{1 / 2}(1+a)-\frac{4}{3} n a^{3 / 2}, 4 n a^{1 / 2}(1+a)^{1 / 2}, 1,-(1+a)^{1 / 2}\right) \\
& =: \chi_{\partial}\left(\bar{y}_{n}, \bar{t}_{n}, 1,-(1+a)^{1 / 2}\right),
\end{aligned}
$$


where we set $\bar{y}_{n}=4 n a^{1 / 2}(1+a)-\frac{4}{3} n a^{3 / 2}$ and $\bar{t}_{n}=4 n a^{1 / 2}(1+a)^{1 / 2}$. If $n a^{1 / 2}$ belongs to a small but fixed neighborhood of 0 of size $Y$, we can write the right hand side in the last equation as

$$
\chi_{\partial}\left(\bar{y}_{n}, \bar{t}_{n}, 1,-(1+a)^{1 / 2}\right)=\nabla_{y, t} \chi_{\partial}\left(\bar{y}, \bar{t}, 1,-(1+a)^{1 / 2}\right)\left(\begin{array}{c}
\bar{y}_{n} \\
\bar{t}_{n}
\end{array}\right),
$$

for some $(\bar{y}, \bar{t}) \in\left[0, \bar{y}_{n}\right] \times\left[0, \bar{t}_{n}\right]$. The matrix $\nabla_{y, t} \chi_{\partial}\left(\bar{y}, \bar{t}, 1,-(1+a)^{1 / 2}\right)$ is independent of $\bar{t}$ (since it is given by the inverse of $\nabla_{y, t}\left(\chi_{\partial}^{-1}\right)$ computed in (3.28)) and at $\bar{y}=0$ it is close to the identity, therefore if $\bar{y}_{n}$ belongs to a small, fixed neighborhood of 0 of size $y_{0}$, the matrix $\nabla_{y, t} \chi_{\partial}\left(\bar{y}, \bar{t}, 1,-(1+a)^{1 / 2}\right)$ remains close to the identity.

We can now estimate the number of iterations $N$ that we will use in our construction. Since $\bar{y}_{n}=4 n a^{1 / 2}+O\left(n a^{3 / 2}\right)$, choose $N \geq 1$ such that $N a^{1 / 2}$ is so small that for $0 \leq \bar{y} \leq \bar{y}_{N}$ the gradient matrix $\nabla_{y, t}\left(\chi_{\partial}\right)\left(\bar{y}, \cdot, 1,-(1+a)^{1 / 2}\right)$ is close to the identity. This is possible due to the independence from $\bar{t}_{N}, \bar{t}$ of the gradient matrix and its uniform boundedness. Since from the initial assumption we have $\left|b^{2 / 3}(y)-1\right|<1 / 10$ for $y \in$ $[0, Y]$, we can take

$$
4 N \simeq C_{0} Y a^{-1 / 2}
$$

for some sufficiently small constant $0<C_{0} \leq 1$.

Remark 3.27. The operator $J$ is elliptic in a small, fixed neighborhood of 0 . If we take $Y$ smaller if necessary, the symbol $d$ of $J$ will be elliptic for $y \in[0, Y]$, therefore the symbol of $J \circ \operatorname{Tr}_{ \pm}\left(u_{F, h}^{n}\right)$ will remain elliptic for any $0 \leq n \leq N$ with $N$ given by (3.33) if $C_{0}$ is small enough. This will be useful when computing the $L^{r}$ norms of the cusps $u_{h}^{n}$, whose symbols will depend on $d$ and therefore will be elliptic uniformly in $0 \leq n \leq N$.

In the following we set

$$
a:=\frac{\sqrt{C_{0}}}{2} Y^{1 / 2} h^{(1-\epsilon) / 2} .
$$

In the remaining part of this section we estimate the time interval $[0, T]$ on which the norm of $U_{h}$ will be evaluated. Using Proposition 3.28 below and $N$ as in (3.33) we see that for $0 \leq n \leq N$, the cusp $u_{h}^{n}(\cdot, t)$ is essentially supported for $(x, y, t)$ such that

$$
\partial_{\tau} \theta\left(x, y, t, 1,-(1+a)^{1 / 2}\right) \in I_{n}\left(c_{0}\right),
$$

where

$$
I_{n}\left(c_{0}\right)=2 a^{1 / 2}(1+a)^{1 / 2} \times\left[2 n-\left(1+c_{0}\right), 2 n+\left(1+c_{0}\right)\right] .
$$

We shall choose $T$ to belong to the essential support of $u_{h}^{N}$. From Proposition 3.28 below it follows that on the essential support of $u_{h}^{N}$ the following should hold:

$$
\begin{aligned}
\left(\partial_{\eta} \theta-(1+a)^{1 / 2} \partial_{\tau} \theta\right)(x, y, t, 1 & \left.-(1+a)^{1 / 2}\right) \\
& =-\frac{4}{3} N a^{3 / 2} \pm \frac{2}{3}(-\zeta)^{3 / 2}\left(x, y, 1,-(1+a)^{1 / 2}\right)
\end{aligned}
$$

We introduce the defining function for the caustic set, denoted $C(y, \eta, \tau)$, as follows:

$$
-\zeta(x, y, \eta, \tau)=0 \quad \text { if and only if } \quad x=C(y, \eta, \tau) .
$$


By the linearity of the phase $\theta(x, y, t, \eta, \tau)$ in time, it follows that the map

$$
y \mapsto \partial_{\eta} \theta\left(C\left(y, 1,-(1+a)^{1 / 2}\right), y, \cdot 1,-(1+a)^{1 / 2}\right)
$$

is independent of time. Moreover, (3.35) is a diffeomorphism in a neighborhood of $y=0$ since its derivative does not vanish.

Let $\bar{t}_{N}:=4 N a^{1 / 2}(1+a)^{1 / 2}$ be the center of the interval $I_{N}\left(c_{0}\right)$. From the discussion above (and if $C_{0}$ in (3.33) is small) it follows that there exists a unique $y_{N} \in[0, Y]$ with

$$
\partial_{\eta} \theta\left(C\left(y_{N}, 1,-(1+a)^{1 / 2}\right), y_{N}, \cdot, 1,-(1+a)^{1 / 2}\right)=(1+a)^{1 / 2} \bar{t}_{N}-\frac{4}{3} N a^{3 / 2}=\bar{y}_{N} \text {. }
$$

We can now define $T$ as the unique time value which satisfies

$$
\partial_{\tau} \theta\left(C\left(y_{N}, 1,-(1+a)^{1 / 2}\right), y_{N}, T, 1,-(1+a)^{1 / 2}\right)=\bar{t}_{N} .
$$

3.4.2. Localization properties of $u_{h}^{n}$. We now describe the essential supports of $u_{h}^{n}$ and show that they are contained in almost disjoint intervals obtained by taking the image of $I_{n}\left(c_{0}\right)$ defined in Proposition 3.18 under the symplectomorphism $\chi_{\partial}$.

Proposition 3.28. We have

$$
\begin{aligned}
\operatorname{ess}-\operatorname{supp}\left(u_{h}^{n}\right) \subset & \left\{(x, y, t) \mid \partial_{\tau} \theta\left(x, y, t, 1,-(1+a)^{1 / 2}\right) \in I_{n}\left(c_{0}\right)\right. \text { and } \\
& \left.\left(\partial_{\eta} \theta-(1+a)^{1 / 2} \partial_{\tau} \theta+\frac{4}{3} n a^{3 / 2} \mp \frac{2}{3}(-\zeta)^{3 / 2}\right)\left(x, y, t, 1,-(1+a)^{1 / 2}\right)=0\right\} .
\end{aligned}
$$

By ess-supp $\left(u_{h}^{n}\right)$ we denote the closure of the set outside of which $u_{h}^{n}$ is $O_{L^{2}}\left(h^{\infty}\right)$.

Proof. The proposition follows using Proposition 3.19, which gives information about the essential support of the symbol $g_{h}^{n}(\cdot, y, \eta) \in \mathcal{S}_{\left[-c_{0}, c_{0}\right]}(\lambda / n)$. Let $c \in(0,1)$ be such that

$$
\left|\frac{\partial_{\tau} \theta\left(x, y, t, \eta,-\eta(1+a)^{1 / 2}\right)}{2(1+a)^{1 / 2} a^{1 / 2}}-2 n\right| \geq 1+c .
$$

It is enough to show that on the essential support of $u_{h}^{n}$ we must have $c \leq c_{0}$, which follows from Proposition 3.24.

Lemma 3.29. Let

$$
J_{n}:=\left\{t \mid \exists(x, y, t) \in \operatorname{ess}-\operatorname{supp}\left(u_{h}^{n}\right), \partial_{\tau} \theta\left(x, y, t, 1,-(1+a)^{1 / 2}\right) \in I_{n}\left(c_{0} / 3-1\right)\right\}
$$

and let $\left|J_{n}\right|$ denote its size. Then

$$
\left|J_{n}\right| \geq c_{0} a^{1 / 2}
$$

Moreover, if $c_{0}$ is sufficiently small and if $(x, y, t)$ is such that $t \in J_{n}$, then

$$
\frac{1}{2} a \leq x \leq \frac{3}{2} a
$$


The proof of Lemma 3.29 uses arguments similar to those in the proof of Proposition 3.28 and is left to the reader. In the same way we can show the following:

Lemma 3.30. Let $k \geq 0$ and $t \in J_{k}$. Then

$$
U_{h}(x, y, t)=u_{h}^{k}(x, y, t)+O_{L^{2}}\left(h^{\infty}\right) .
$$

Remark 3.31. Lemma 3.30 shows that $u_{h}^{n}$ have almost disjoint essential supports in the variables $(y, t)$. Therefore, Proposition 3.23 applies to show that $U_{h}$ defined in (3.26) is also an approximate solution to (2.4) in the sense that for $t \in[0, T]$ we have

$$
\left\|\square_{g} U_{h}(\cdot, t)\right\|_{L^{2}(\Omega)} \leq O\left(h^{-1}\right)\left\|U_{h}(\cdot, t)\right\|_{L^{2}(\Omega)} .
$$

In the rest of this section we prove that $U_{h}$ satisfies the Dirichlet boundary condition.

Proposition 3.32. The approximate solution $U_{h}$ to (2.4) defined in (3.26) satisfies

$$
\left.U_{h}\right|_{\partial \Omega \times[0, T]}=O\left(h^{\infty}\right) .
$$

Proof. From Proposition 3.22 we deduce

$$
U_{h}(0, y, t)=J\left(\operatorname{Tr}_{+}\left(u_{F, h}^{0}\right)\right)(y, t)+J\left(\operatorname{Tr}_{-}\left(u_{F, h}^{N}\right)\right)(y, t) .
$$

The first term in the right hand side of (3.41) is essentially supported for $t$ in a small interval that does not meet $[0, T]$, hence its contribution is clearly trivial for $t \in[0, T]$. We now deal with the second term in (3.41): it will be enough to show that

$$
T \notin \operatorname{ess}-\operatorname{supp}\left(J\left(\operatorname{Tr}_{-}\left(u_{F, h}^{N}\right)\right)\right) .
$$

We argue by contradiction and assume that (3.42) fails to hold. Then on the support of $g_{h}^{N}$ we must have, for some $y_{T} \in[0, Y]$,

$$
\left|\frac{\left(\partial_{\tau} \theta_{0}+\xi \partial_{\tau} \zeta_{0}\right)\left(y_{T}, T, 1,-(1+a)^{1 / 2}\right)}{2(1+a)^{1 / 2} a^{1 / 2}}-2 n\right| \leq c_{0} .
$$

From Proposition 3.24 it follows that $\xi=-\left(-\zeta_{0}\right)^{1 / 2}\left(1,-(1+a)^{1 / 2}\right)=-a$ in $(3.43)$. Replacing this in (3.43) and using $\partial_{\tau} \zeta_{0}=2(1+a)^{1 / 2}+O(a)$ we find that

$$
\partial_{\tau} \theta_{0}\left(y_{T}, T, 1,-(1+a)^{1 / 2}\right) \in 2 a^{1 / 2}(1+a)^{1 / 2}\left[2 N+1-c_{0}, 2 N+1+c_{0}\right] .
$$

From Proposition 3.28 we must also have

$$
\begin{aligned}
\partial_{\eta} \theta_{0}\left(y_{T}, T, 1,-(1+a)^{1 / 2}\right)= & (1+a)^{1 / 2} \partial_{\tau} \theta_{0}\left(y_{T}, T, 1,-(1+a)^{1 / 2}\right) \\
& -\frac{4}{3} N a^{3 / 2}-\frac{2}{3}\left(-\zeta_{0}\right)^{3 / 2}\left(1,-(1+a)^{1 / 2}\right) .
\end{aligned}
$$

From the choice of $T$ in (3.37) we will get a contradiction. Precisely, using (3.37) and (3.36) yields

$$
\begin{aligned}
& \partial_{\tau} \theta_{0}\left(y_{N}, T, 1,-(1+a)^{1 / 2}\right)=\bar{t}_{N}+O(a), \\
& \partial_{\eta} \theta_{0}\left(y_{N}, T, 1,-(1+a)^{1 / 2}\right)=\bar{y}_{N}+O(a),
\end{aligned}
$$


where we recall that $\bar{T}_{N}=4 N a^{1 / 2}(1+a)^{1 / 2}$ and $\bar{y}_{N}=(1+a)^{1 / 2} \bar{t}_{N}-\frac{4}{3} N a^{3 / 2}$. Notice also that for $N$ defined by (3.33) the contribution of $N a^{3 / 2}$ is $O(a)$.

Using (3.44)-(3.47) now gives

$$
\begin{gathered}
\left|\partial_{\eta} \theta_{0}\left(y_{T}, T, 1,-(1+a)^{1 / 2}\right)-\partial_{\eta} \theta_{0}\left(y_{N}, T, 1,-(1+a)^{1 / 2}\right)\right| \\
=(1+a)^{1 / 2}\left|\partial_{\tau} \theta_{0}\left(y_{T}, T, 1,-(1+a)^{1 / 2}\right)-\partial_{\tau} \theta_{0}\left(y_{N}, T, 1,-(1+a)^{1 / 2}\right)\right|+O(a) \\
\in\left[2 a^{1 / 2}(1+a)^{1 / 2}\left(1-c_{0}\right), 2 a^{1 / 2}(1+a)^{1 / 2}\left(1+c_{0}\right)\right]
\end{gathered}
$$

(where the inclusion in the last line follows from (3.44) and (3.46)). Evaluating the terms in the first and second lines of (3.48) yields

$$
3\left(1-c_{0}\right) \leq 1+c_{0}
$$

hence $c_{0} \geq 1 / 2$, which is a contradiction since in Section 3.2.1 we have chosen $0<c_{0} \leq 3 / 8$. Therefore (3.42) does hold and in the same way we can see that $\operatorname{ess}-\operatorname{supp}\left(J\left(\operatorname{Tr}_{-}\left(u_{F, h}^{N}\right)\right)\right.$ does not meet the interval $[0, T]$.

3.4.3. Strichartz estimates for the approximate solution $U_{h}$

Proposition 3.33. Let $r>4$ and $\epsilon>0$ be the one fixed in Section 3. Define

$$
\beta(r)=\frac{3}{2}\left(\frac{1}{2}-\frac{1}{r}\right)+\frac{1}{6}\left(\frac{1}{4}-\frac{1}{r}\right)
$$

and let $\beta \leq \beta(r)-\epsilon$. Then the approximate solution $U_{h}$ of the wave equation (2.4) satisfies

$$
h^{\beta}\left\|U_{h}\right\|_{L^{q}\left([0, T], L^{r}(\Omega)\right)} \geq h^{-7 \epsilon / 8}\left\|\left.U_{h}\right|_{t=0}\right\|_{L^{2}(\Omega)} \gg\left\|\left.U_{h}\right|_{t=0}\right\|_{L^{2}(\Omega)} .
$$

Remark 3.34. Notice that the condition $\beta<\beta(r)$ shows that $U_{h}$ cannot satisfy the Strichartz inequalities of the free case, a loss of at least $\frac{1}{6}\left(\frac{1}{4}-\frac{1}{r}\right)$ derivatives being unavoidable.

Proof. The key point here is that the $u_{h}^{n}$ have almost disjoint supports in time and in the tangential variable, hence we can bound from below the $L^{q}([0, T])$ norm by a sum of integrals over small intervals of time $J_{k}$ on which there will be only one cusp $u_{h}^{k}$ to consider, the contribution from all the others being trivial. The intervals $J_{k}$ will be the ones defined in (3.39) for which Lemma 3.29 applies. We have

$$
\begin{aligned}
\left\|U_{h}\right\|_{L^{q}\left([0, T], L^{r}(\Omega)\right)}^{q} & =\int_{0}^{T}\left\|U_{h}(\cdot, t)\right\|_{L^{r}(\Omega)}^{q} d t=\int_{0}^{T}\left\|\sum_{n=0}^{N} u_{h}^{n}(\cdot, t)\right\|_{L^{r}(\Omega)}^{q} d t \\
& \geq \sum_{k \leq N} \int_{J_{k}}\left\|\sum_{n=0}^{N} u_{h}^{n}(\cdot, t)\right\|_{L^{r}(\Omega)}^{q} d t+O\left(h^{\infty}\right) \\
& \simeq \sum_{k \leq N}\left|J_{k}\right|\left\|u_{h}^{0}(\cdot, 0)\right\|_{L^{r}(\Omega)}^{q}+O\left(h^{\infty}\right) \\
& \simeq \frac{c_{0}}{4} Y\left\|u_{h}^{0}(\cdot, 0)\right\|_{L^{r}(\Omega)}^{q}+O\left(h^{\infty}\right) .
\end{aligned}
$$


Indeed, we have shown in Lemma 3.30 that for $t$ belonging to small intervals of time $J_{k}$, only $u_{h}^{k}$ has to be considered in the sum since the contribution from each $u_{h}^{n}$ with $n \neq k$ is $O_{L^{2}}\left(h^{\infty}\right)$. In the last line of (3.50) we used Lemma 3.29 to estimate $\left|J_{k}\right|$ from below, uniformly in $k$, by $c_{0} a^{1 / 2}$, where $c_{0} \in(0,3 / 8]$ is fixed, and the fact that $N \simeq \frac{Y}{4} a^{-1 / 2}$.

For $t \in J_{k}$, it follows from (3.40) that $x \geq a / 2$, therefore on the essential support of $u_{h}^{k}(\cdot, t)$ the normal variable does not approach the boundary. Therefore the restrictions of $u_{h}^{k}$ to $J_{k}$ have disjoint supports.

Using similar arguments to [10, Prop. 6.7] we obtain the following result concerning the $L^{r}$ norms of the phase integrals associated to a cusp type Lagrangian:

Proposition 3.35. Let $N$ be defined by (3.33). For $t \in J_{n}$ defined in (3.39) the $L^{r}(\Omega)$ norms of a cusp $u_{h}^{n}(\cdot, t)$ of the form (3.25) satisfy, uniformly for $n \in\{0, \ldots, N\}$,

(i) for $2 \leq r<4$

$$
\begin{aligned}
\left\|u_{h}^{n}(\cdot, t)\right\|_{L^{r}(\Omega)} & \simeq h^{\frac{1}{r}+\frac{1}{2}} a^{\frac{1}{r}-\frac{1}{4}}, \\
\left\|u_{h}^{n}(\cdot, 0)\right\|_{L^{2}(\Omega)} & \simeq h a^{1 / 4}
\end{aligned}
$$

(ii) for $r>4$,

$$
\left\|u_{h}^{n}(\cdot, t)\right\|_{L^{r}(\Omega)} \simeq h^{\frac{1}{3}+\frac{5}{3 r}} .
$$

Using Proposition 3.35 and (3.34) we deduce that there are constants $C=C(Y)$ independent of $h$ such that for $r=2$,

$$
\left\|\left.U_{h}\right|_{t=0}\right\|_{L^{2}(\Omega)} \simeq h\left\|\left.\partial_{t} U_{h}\right|_{t=0}\right\|_{L^{2}(\Omega)} \simeq\left\|u_{h}^{0}(\cdot, 0)\right\|_{L^{2}(\Omega)} Y^{1 / 8} h^{1+(1-\epsilon) / 8} .
$$

For $r>4$ we get, using (3.50),

$$
\left\|U_{h}\right\|_{L^{q}\left([0, T], L^{r}(\Omega)\right)} \geq C(Y) h^{\frac{1}{3}+\frac{5}{3 r}}, \quad \text { where } \quad C(Y)=\left(\frac{c_{0}}{4} Y\right)^{1 / q} .
$$

We deduce that for $\beta \leq \beta(r)-\epsilon$,

$$
h^{\beta}\left\|U_{h}\right\|_{L^{q}\left([0, T], L^{r}(\Omega)\right)} \geq C(Y) h^{\beta(r)-\epsilon} h^{\frac{1}{3}+\frac{5}{3 r}} \gg\left\|\left.U_{h}\right|_{t=0}\right\|_{L^{2}(\Omega)}+h\left\|\left.\partial_{t} U_{h}\right|_{t=0}\right\|_{L^{2}},
$$

where we recall that $Y$ was fixed, depending on $b$ and, hence, on $\Omega$ only.

3.4.4. End of proof of Theorem 2.2. We can now finish the proof of Theorem 2.2. Let $\epsilon>0$ be as in Section 3 and let $N$ be given by (3.27). Consider the $L^{2}$ normalized approximate solution to (2.4),

$$
v_{h, \epsilon}^{n}(x, y, t):=\frac{1}{\left\|U_{h}(\cdot, 0)\right\|_{L^{2}(\Omega)}} u_{h}^{n}(x, y, t),
$$

and set

$$
\tilde{V}_{h, \epsilon}(x, y, t):=\sum_{n=0}^{N} v_{h}^{n}(x, y, t)=\frac{1}{\left\|U_{h}(\cdot, 0)\right\|_{L^{2}(\Omega)}} U_{h}(x, y, t) .
$$


We claim that $\tilde{V}_{h, \epsilon}$ and $v_{h}^{n}$ satisfy the conditions of Theorem 2.2. Indeed, it follows from Proposition 3.35 that for $4<r<\infty, v_{h, \epsilon}^{n}$ satisfy

$$
\left\{\begin{array}{l}
\left\|v_{h, \epsilon}^{n}(\cdot, t)\right\|_{L^{r}(\Omega)} \geq C h^{-\frac{3}{2}\left(\frac{1}{2}-\frac{1}{r}\right)-\frac{1}{6}\left(\frac{1}{4}-\frac{1}{r}\right)+2 \epsilon} \quad \text { for } t \in J_{n}, \\
\sup _{\epsilon>0}\left\|v_{h, \epsilon}^{n}(\cdot, t)\right\|_{L^{2}(\Omega)} \leq 1,
\end{array}\right.
$$

where in order to bound the $L^{2}$ norms uniformly we use the fact that for $t \in J_{n}$,

$$
\left\|u_{h}^{n}(\cdot, t)\right\|_{L^{2}(\Omega)} \simeq\left\|u_{h}^{0}(\cdot, t)\right\|_{L^{2}(\Omega)} \simeq\left\|u_{h}^{0}(\cdot, 0)\right\|_{L^{2}(\Omega)}=\left\|U_{h}(\cdot, 0)\right\|_{L^{2}(\Omega)} .
$$

From Proposition 3.28, the cusps $v_{h, \epsilon}^{n}$ have almost disjoint essential supports in the time and tangential variables and for the normal variable in an interval of size $a \simeq$ $\frac{1}{2} Y^{1 / 2} h^{(1-\epsilon) / 2}$. Moreover, the approximate solution $\tilde{V}_{h, \epsilon}$ is localized at spatial frequency $1 / h$ and satisfies

$$
\left\|\tilde{V}_{h, \epsilon}\right\|_{L^{2}(\Omega)} \lesssim 1, \quad\left\|\partial_{y} \tilde{V}_{h, \epsilon}\right\|_{L^{2}(\Omega)} \lesssim \frac{1}{h}, \quad\left\|\partial_{y}^{2} \tilde{V}_{h, \epsilon}\right\|_{L^{2}(\Omega)} \lesssim \frac{1}{h^{2}},
$$

with constants independent of $\epsilon$, which follows from the spectral localization together with the uniform bounds of the derivatives of $g_{h}^{n}$ with respect to $y$. From Proposition 3.23 and the almost orthogonality property of the supports in $y$ we also obtain

$$
\square_{g} \tilde{V}_{h, \epsilon}=O_{L^{2}(\Omega)}(1 / h) .
$$

Proposition 3.32 ensures that the Dirichlet condition holds on $\Omega \times[0, T]$.

Acknowledgments. The author would like to thank Gilles Lebeau who suggested this problem to her and Nicolas Burq for many helpful discussions on the subject. She would also like to thank Michael Taylor for having sent her the manuscript "Boundary problems for the wave equations with grazing and gliding rays".

This research was partly supported by the A.N.R. grant 07-BLAN-0250.

\section{References}

[1] Blair, M. D., Smith, H. F., Sogge, C. D.: Strichartz estimates for the wave equation on manifolds with boundary. Ann. Inst. H. Poincaré Anal. Non Linéaire 26, 1817-1829 (2009) Zbl 1198.58012 MR 2566711

[2] Bouclet, J.-M., Tzvetkov, N.: On global Strichartz estimates for non-trapping metrics. J. Funct. Anal. 254, 1661-1682 (2008) Zbl 1168.35005 MR 2396017

[3] Burq, N., Lebeau, G., Planchon, F.: Global existence for energy critical waves in 3-D domains. J. Amer. Math. Soc. 21, 831-845 (2008) Zbl 1204.35119 MR 2393429

[4] Burq, N., Planchon, F.: Global existence for energy critical waves in 3-d domains : Neumann boundary conditions. Amer. J. Math. 131, 1715-1742 (2009) Zbl 1184.35210 MR 2567505

[5] Cazenave, T., Weissler, F. B.: The Cauchy problem for the critical nonlinear Schrödinger equation in $H^{s}$. Nonlinear Anal. 14, 807-836 (1990) Zbl 0706.35127 MR 1055532

[6] Eskin, G.: Parametrix and propagation of singularities for the interior mixed hyperbolic problem. J. Anal. Math. 32, 17-62 (1977) Zbl 0375.35037 MR 0477491 
[7] Ginibre, J., Velo, G.: The global Cauchy problem for the nonlinear Schrödinger equation revisited. Ann. Inst. H. Poincaré Anal. Non Linéaire 2, 309-327 (1985) Zbl 0586.35042 MR 0801582

[8] Ginibre, J., Velo, G.: Generalized Strichartz inequalities for the wave equation. In: Partial Differential Operators and Mathematical Physics (Holzhau, 1994), Oper. Theory Adv. Appl. 78, Birkhäuser, Basel, 153-160 (1995) Zbl 0839.35016 MR 1365328

[9] Hörmander, L.: The Analysis of Linear Partial Differential Operators. III. Grundlehren Math. Wiss. 274, Springer, Berlin (1985) Zbl 0601.35001 MR 1313500

[10] Ivanovici, O.: Counterexamples to Strichartz estimates for the wave equation in domains. Math. Ann. 347, 627-673 (2010) Zbl 1201.35060 MR 2640046

[11] Ivanovici, O., Lebeau, G., Planchon, F.: Dispersive estimates for the wave equation in strictly convex domains. Preprint (2010)

[12] Kapitanski1̌, L. V.: Estimates for norms in Besov and Lizorkin-Triebel spaces for solutions of second-order linear hyperbolic equations. Zap. Nauchn. Sem. Leningrad. Otdel. Mat. Inst. Steklov. (LOMI) 171, 106-162, 185-186 (1989) (in Russian) MR 1031987

[13] Kapitanski1̌, L. V.: Some generalizations of the Strichartz-Brenner inequality. Algebra i Analiz 1, no. 3, 127-159 (1989) (in Russian) Zbl 0732.35118 MR 1015129

[14] Kato, T.: On nonlinear Schrödinger equations. Ann. Inst. H. Poincaré Phys. Théor. 46, 113 129 (1987) Zbl 0632.35038 MR 0877998

[15] Keel, M., Tao, T.: Endpoint Strichartz estimates. Amer. J. Math. 120, 955-980 (1998) Zbl 0922.35028 MR 1646048

[16] Lebeau, G.: Estimation de dispersion pour les ondes dans un convexe. In: Journées "Équations aux Dérivées Partielles" (Evian, 2006); http://jedp.cedram.org/item?id= JEDP_2006_-__A7_0.

[17] Lindblad, H., Sogge, C. D.: On existence and scattering with minimal regularity for semilinear wave equations. J. Funct. Anal. 130, 357-426 (1995) Zbl 0846.35085 MR 1335386

[18] Melrose, R. B.: Equivalence of glancing hypersurfaces. Invent. Math. 37, 165-191 (1976) Zbl 0354.53033 MR 0436225

[19] Mockenhaupt, G., Seeger, A., Sogge, C. D.: Local smoothing of Fourier integral operators and Carleson-Sjölin estimates. J. Amer. Math. Soc. 6, 65-130 (1993) Zbl 0776.58037 MR 1168960

[20] Ralston, J. V.: Approximate eigenfunctions of the Laplacian. J. Differential Geom. 12, 87-100 (1977) Zbl 0385.58012 MR 0470998

[21] Smith, H. F.: A parametrix construction for wave equations with $C^{1,1}$ coefficients. Ann. Inst. Fourier (Grenoble) 48, 797-835 (1998) Zbl 0974.35068 MR 1644105

[22] Smith, H. F., Sogge, C. D.: On the critical semilinear wave equation outside convex obstacles. J. Amer. Math. Soc. 8, 879-916 (1995) Zbl 0860.35081 MR 1308407

[23] Smith, H. F., Sogge, C. D.: On the $L^{p}$ norm of spectral clusters for compact manifolds with boundary. Acta Math. 198, 107-153 (2007) Zbl 1189.58017 MR 2316270

[24] Staffilani, G., Tataru, D.: Strichartz estimates for a Schrödinger operator with nonsmooth coefficients. Comm. Partial Differential Equations 27, 1337-1372 (2002) Zbl 1010.35015 MR 1924470

[25] Strichartz, R. S.: Restrictions of Fourier transforms to quadratic surfaces and decay of solutions of wave equations. Duke Math. J. 44, 705-714 (1977) Z Zbl 0372.35001 MR 0512086

[26] Tataru, D.: Strichartz estimates for second order hyperbolic operators with nonsmooth coefficients. III. J. Amer. Math. Soc. 15, 419-442 (2002) Zbl 0990.35027 MR 1887639

[27] Vasy, A.: Propagation of singularities for the wave equation on manifolds with corners. Ann. of Math. (2) 168, 749-812 (2008) Zbl 1171.58007 MR 2456883 